\title{
A New, Powerful Glycosylation Method: Activation of Thioglycosides with Dimethyl Disulfide-Triflic Anhydride
}

\author{
János Tatai and Péter Fügedi* \\ Chemical Research Center, Hungarian Academy of Sciences, \\ Pusztaszeri út 59-67, H-1025 Budapest, Hungary
}

\section{Supporting Material}

\section{General methods:}

All reactions were performed in flame dried flasks fitted with a rubber septum under a positive pressure of argon. Air- and moisture-sensitive liquids and solutions were transferred via syringe. Molecular sieves $(4 \AA)$ were flame dried before use. Dimethyl disulfide and trifluoromethanesulfonic anhydride were purchased from Fluka. Dichloromethane was distilled from $\mathrm{CaH}_{2}$, and stored over $4 \AA$ molecular sieves. Diethyl ether (p.a., E. Merck) and acetonitrile (p.a., E. Merck) were stored over $4 \AA \AA$ molecular sieves before use. Solvents used for column chromatography were of technical grade and distilled before use. Thin layer chromatography (TLC) was performed on silica gel $60 \mathrm{~F}_{254}$ plates (E. Merck, Darmstadt), the compounds were detected under UV light and by spraying the plates with a $0.02 \mathrm{M}$ solution of resorcinol in $20 \%$ methanolic $\mathrm{H}_{2} \mathrm{SO}_{4}$ solution followed by heating. For column chromatography silica gel 60 (0.040-0.063 mm) (E. Merck) was employed.

Melting points were determined in capillary tubes on a Griffin melting point apparatus and are uncorrected. Optical rotations were measured at $23{ }^{\circ} \mathrm{C}$ with an Optical Activity AA-10R polarimeter. The NMR spectra were recorded on Varian Unity Inova $4000{ }^{1} \mathrm{H}$ : $400 \mathrm{MHz}$; ${ }^{13} \mathrm{C}$ : $\left.100 \mathrm{MHz}\right)$ Varian Unity Inova $3000\left({ }^{1} \mathrm{H}: 300 \mathrm{MHz} ;{ }^{13} \mathrm{C}: 75 \mathrm{MHz}\right)$ and Varian Unity Inova $2000\left({ }^{1} \mathrm{H}: 200 \mathrm{MHz} ;{ }^{13} \mathrm{C}: 50 \mathrm{MHz}\right)$ spectrometers at ambient temperature in $\mathrm{CDCl}_{3}$, and assigned using 2D-methods (COSY, HSQC). The chemical shifts were referenced to TMS $\left(0.00 \mathrm{ppm}\right.$ for $\left.{ }^{1} \mathrm{H}\right)$ and to the central line of $\mathrm{CDCl}_{3}\left(77.0 \mathrm{ppm}\right.$ for $\left.{ }^{13} \mathrm{C}\right)$ as internal standards. Elemental analyses were performed with an Elementar Vario EL III instrument at the Analytical Department of the Chemical Research Center, Hungarian Academy of Sciences. 


\section{$\mathrm{Me}_{2} \mathrm{~S}_{2}-\mathrm{Tf}_{2} \mathrm{O}$ reagent}

A $1 \mathrm{M}$ solution of the reagent was made by adding trifluoromethanesulfonic anhydride $(0.168$ $\mathrm{mL}, 1 \mathrm{mmol})$ to a solution of dimethyl disulfide $(0.10 \mathrm{~mL}, 1.1 \mathrm{mmol})$ in dry $\mathrm{CH}_{2} \mathrm{Cl}_{2}(0.75$ $\mathrm{mL}$ ) at $0{ }^{\circ} \mathrm{C}$ and stirring the mixture for $30 \mathrm{~min}$ at the same temperature before use. The yellowish brown colored mixture darkened on storage in a freezer, but it retained most of its activating capability after several days.

\section{General method for glycosylation:}

A $1 \mathrm{M}$ solution of the promoter $(0.9 \mathrm{~mL}, 0.9 \mathrm{mmol})$ in $\mathrm{CH}_{2} \mathrm{Cl}_{2}$ was added to a mixture containing the thioglycoside donor $(0.6 \mathrm{mmol})$, the glycosyl acceptor $(0.5 \mathrm{mmol})$, and activated $4 \AA$ powdered molecular sieves $(1 \mathrm{~g})$ in the solvent $(5 \mathrm{~mL})$ indicated. The reaction mixture was stirred for $10 \mathrm{~min}$, quenched by the addition of excess triethylamine $(1 \mathrm{~mL}, 7.2$ mmol), diluted with $\mathrm{CH}_{2} \mathrm{Cl}_{2}(100 \mathrm{~mL})$, filtered, and washed sequentially with $2 \mathrm{M}$ aqueous $\mathrm{HCl}$, saturated aqueous $\mathrm{NaHCO}_{3}$, and water. The organic layer was dried over $\mathrm{MgSO}_{4}$, concentrated under diminished pressure at $40{ }^{\circ} \mathrm{C}$, and the residue was purified by column chromatography.

Procedure $a$ : solvent: $\mathrm{CH}_{2} \mathrm{Cl}_{2}$; reaction temperature: $0{ }^{\circ} \mathrm{C}$.

Procedure $b$ : solvent: $\mathrm{CH}_{2} \mathrm{Cl}_{2}$; $\quad$ reaction temperature: $-40{ }^{\circ} \mathrm{C}$.

Procedure $c$ : solvent: $\mathrm{CH}_{2} \mathrm{Cl}_{2}$; $\quad$ reaction temperature: $-40{ }^{\circ} \mathrm{C}$;

the reaction was performed in the presence of 2,6-di-tert-butyl-4methylpyridine $(0.10 \mathrm{~g}, 0.50 \mathrm{mmol})$.

Procedure $d$ : solvent: $\mathrm{Et}_{2} \mathrm{O}-\mathrm{CH}_{2} \mathrm{Cl}_{2}(5: 2) ; \quad$ reaction temperature: $-40{ }^{\circ} \mathrm{C}$.

Procedure $e$ : solvent: $\mathrm{MeCN}-\mathrm{CH}_{2} \mathrm{Cl}_{2}(5: 2)$; $\quad$ reaction temperature: $-40{ }^{\circ} \mathrm{C}$. 

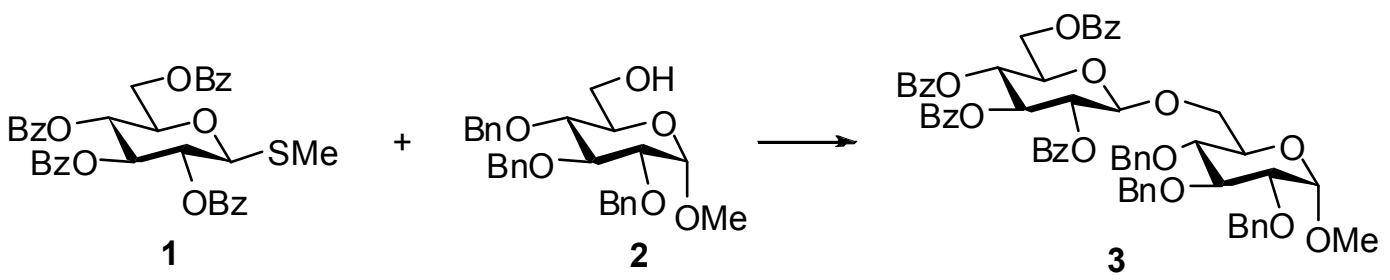

Reaction of $\mathbf{1}^{1}$ with $2^{2}$ by procedure $a$ afforded $3(93 \%)$ as a syrup; $[\alpha]_{\mathrm{D}}+23\left(c 0.5, \mathrm{CHCl}_{3}\right)$, lit. $^{3}[\alpha]_{\mathrm{D}}+18\left(c 1.4, \mathrm{CHCl}_{3}\right)$, lit. $^{4}[\alpha]_{\mathrm{D}}+21.7\left(c\right.$ 1.0, $\left.\mathrm{CHCl}_{3}\right)$. The spectral data were consistent with those in the literature ${ }^{3,4}$.

Methyl (2,3,4,6-tetra- $O$-benzoyl- $\beta$-D-glucopyranosyl)-(1 $\rightarrow 4)-3-O$-benzyl-2benzyloxycarbonylamino-2-deoxy-6-O-(4-methoxy)phenyl- $\alpha$-D-glucopyranoside (5)

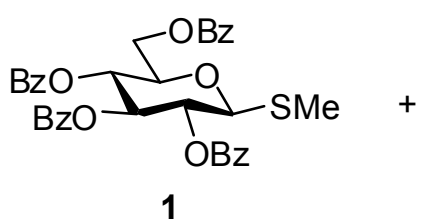

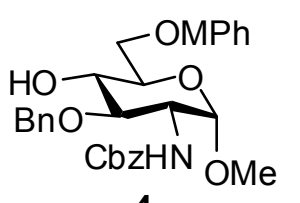

4

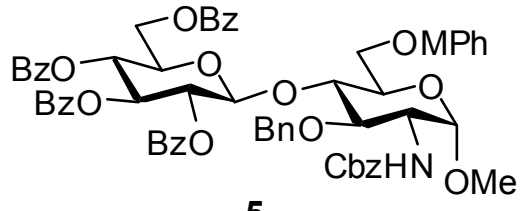

5

Reaction of $\mathbf{1}^{1}$ with $4^{5}$ by procedure $b$ afforded 5 (79\%); mp 113-114 ${ }^{\circ} \mathrm{C}$ (from ethyl acetatehexanes); $[\alpha]_{\mathrm{D}}+61\left(c \mathrm{0.7}, \mathrm{CHCl}_{3}\right) ;{ }^{1} \mathrm{H} \mathrm{NMR}\left(300 \mathrm{MHz}, \mathrm{CDCl}_{3}\right): \delta 7.98(\mathrm{~d}, 2 \mathrm{H}$, aromatic), $7.91(\mathrm{~d}, 2 \mathrm{H}$, aromatic), $7.84(\mathrm{~d}, 2 \mathrm{H}$, aromatic), $7.76(\mathrm{~d}, 2 \mathrm{H}$, aromatic), 7.56-7.15 (m, 22H, aromatic), 6.88 (d, 2H, aromatic), 6.79 (d, 2H, aromatic), 5.65 (t, 1H, $J_{3^{\prime}, 4}, 9.1 \mathrm{~Hz}, \mathrm{H}-3$ '), 5.62 (t, 1H, $J_{4}, 5,9.6 \mathrm{~Hz}, \mathrm{H}-4$ '), 5.55 (t, 1H, $J_{2}, 3,9.3 \mathrm{~Hz}, \mathrm{H}-2$ '), 5.08 (d, 1H, J $11.6 \mathrm{~Hz}, \mathrm{PhCH}_{2}$ ), 5.04 (d, 1H, J 12.3 Hz, $\mathrm{PhCH}_{2}$ ), 5.02 (d, 1H, J $11.6 \mathrm{~Hz}, \mathrm{PhCH}_{2}$ ), 4.92 (d, 1H, J', $, 7.5 \mathrm{~Hz}, \mathrm{H}-$ 1'), 4.72 (d, 1H, J 9.9 Hz, NH), 4.69 (d, 1H, J 11.3 Hz, PhCH $), 4.67$ (d, 1H, J,2 $3.5 \mathrm{~Hz}, \mathrm{H}-1$ ), 4.49 (dd, 1H, $\left.J_{5}, 6 \mathrm{a}^{\prime} 3.3 \mathrm{~Hz}, J_{6 \mathrm{a}^{\prime}, 6 \mathrm{~b}^{\prime}} 10.8 \mathrm{~Hz}, \mathrm{H}-6 \mathrm{a}^{\prime}\right), 4.24$ (dd,1H, $\left.J_{5}{ }^{\prime}, 6 \mathrm{~b}^{\prime}, 4.1 \mathrm{~Hz}, \mathrm{H}-6 \mathrm{~b}^{\prime}\right), 4.15$ (t, $\left.1 \mathrm{H}, J_{4,5} 9.3 \mathrm{~Hz}, \mathrm{H}-4\right), 4.05$ (dd, 1H, $\left.J_{5,6 \mathrm{a}} 3.0 \mathrm{~Hz}, J_{6 \mathrm{a}, 6 \mathrm{~b}} 10.3 \mathrm{~Hz}, \mathrm{H}-6 \mathrm{a}\right), 3.96$ (dd, 1H, $J_{2,3} 10.3$ Hz, H-2), 3.96 (d, 1H, H-6b), 3.84 (m, 1H, H-5'), 3.83 (s, 3H, $\mathrm{ArOCH}_{3}$ ), 3.68 (t, 1H, J3,4 9.0 $\mathrm{Hz}, \mathrm{H}-3), 3.67$ (m, 1H, H-5), 3.24 (s, 3H, $\left.\mathrm{OCH}_{3}\right) ;{ }^{13} \mathrm{C} \mathrm{NMR}\left(75 \mathrm{MHz}, \mathrm{CDCl}_{3}\right): \delta 166.0$

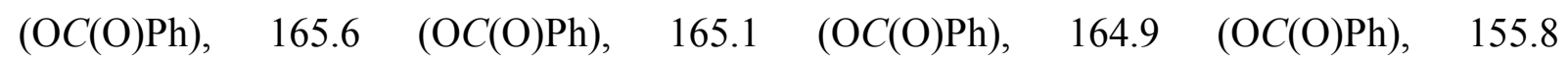
$\left(\mathrm{NHC}(\mathrm{O}) \mathrm{OCH}_{2} \mathrm{Ph}\right), 154.3,152.5,138.6,136.3,133.4,133.3,133.2,133.0,129.8,129.6$, $129.5,128.9,128.7,128.5,128.3,128.2,128.1,127.6,127.3,115.8,114.9$ (aromatic), 100.9 (C-1'), 98.8 (C-1), 78.3 (C-3), 78.1 (C-4), $74.6\left(\mathrm{PhCH}_{2}\right), 73.2$ (C-3'), 72.4 (C-4'), 72.2 (C-5'), 69.5 (C-2'), 69.4 (C-5), $66.8\left(\mathrm{NHC}(\mathrm{O}) \mathrm{OCH}_{2} \mathrm{Ph}\right), 66.5$ (C-6), $62.9\left(\mathrm{C}-6\right.$ '), $55.7\left(\mathrm{ArOCH}_{3}\right)$, 
$55.2\left(\mathrm{OCH}_{3}\right), 54.5$ (C-2); Anal. Calcd for $\mathrm{C}_{63} \mathrm{H}_{59} \mathrm{NO}_{17}: \mathrm{C}, 68.66 ; \mathrm{H}, 5.40 ; \mathrm{N}, 1.27$. Found: $\mathrm{C}$, $68.49 ; \mathrm{H}, 5.42 ; \mathrm{N}, 1.26$.

Methyl $(2,3,4,6$-tetra- $O$-benzoyl- $\beta$-D-glucopyranosyl)-(1 $\rightarrow 4)-2,3,6$-tri- $O$-benzyl- $\alpha$-Dglucopyranoside (7)

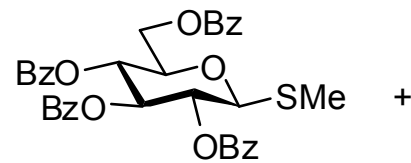

1<smiles>COC(OCc1ccccc1)C(O)C(O)COc1ccccc1</smiles>

6

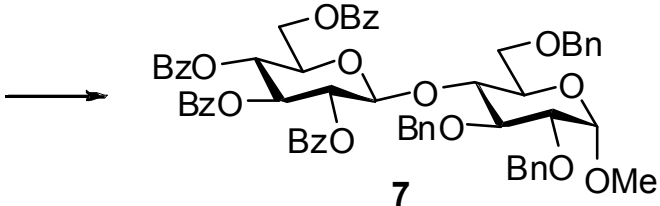

7

Reaction of $\mathbf{1}^{1}$ with $\mathbf{6}^{6}$ by procedure $b$ afforded $7(67 \%)$ as a syrup; $[\alpha]_{\mathrm{D}}-3\left(c 0.5, \mathrm{CHCl}_{3}\right)$, lit. $^{3}$ $[\alpha]_{\mathrm{D}}+1\left(c 1.5, \mathrm{CHCl}_{3}\right)$, lit. $^{7}[\alpha]_{\mathrm{D}}-3\left(c 1.0, \mathrm{CHCl}_{3}\right)$. The spectral data were consistent with those in the literature ${ }^{3}$.

Methyl (2,3,4,6-tetra- $O$-benzoyl- $\beta$-D-glucopyranosyl)-( $1 \rightarrow 3)-2-O$-benzyl-4,6-O-

benzylidene- $\alpha$-D-glucopyranoside (9)

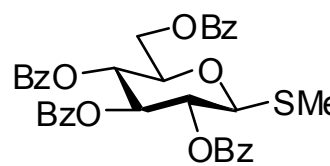

1

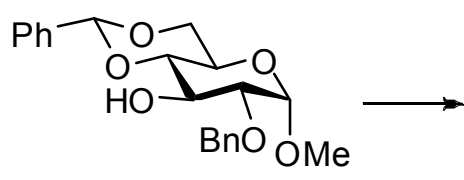

8

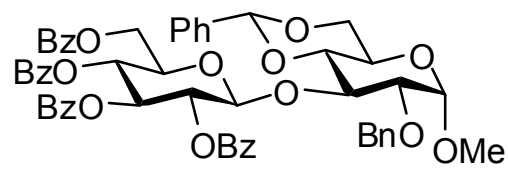

9

Reaction of $\mathbf{1}^{1}$ with $\mathbf{8}^{8}$ by procedure $c$ afforded 9 (76\%); mp 155-156 ${ }^{\circ} \mathrm{C}$, lit. ${ }^{9} \mathrm{mp} 180-182{ }^{\circ} \mathrm{C}$; $[\alpha]_{\mathrm{D}}-21\left(c 0.5, \mathrm{CHCl}_{3}\right)$, lit. $^{9}[\alpha]_{\mathrm{D}}-21\left(c 1.0, \mathrm{CHCl}_{3}\right)$. The spectral data were in agreement with those in the literature 9 .

Methyl (2,3,4,6-tetra- $O$-benzoyl- $\beta$-D-glucopyranosyl)-(1 $\rightarrow 2)-3-O$-benzyl-4,6-Obenzylidene- $\alpha$-D-glucopyranoside (11)

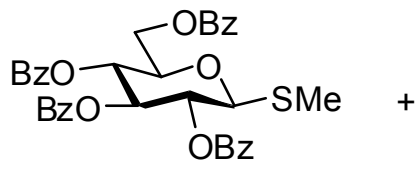

1

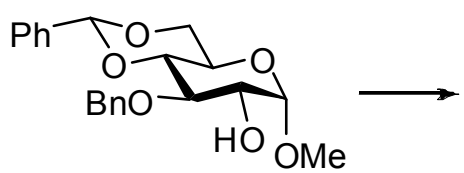

10

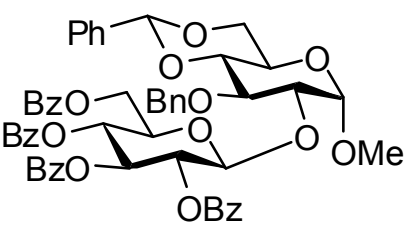

11 
Reaction of $\mathbf{1}^{1}$ with $\mathbf{1 0}^{8}$ by procedure $c$ afforded 11 (93\%); mp 113-114 ${ }^{\circ} \mathrm{C}$ (from ethyl acetate-hexanes); $[\alpha]_{\mathrm{D}}+16\left(c 0.9, \mathrm{CHCl}_{3}\right)$, lit. $^{10}[\alpha]_{\mathrm{D}}+18\left(c 1.6, \mathrm{CHCl}_{3}\right)$. The spectral data were consistent with those in the literature ${ }^{11}$.

Methyl (2,3,6-tri-O-acetyl-2-deoxy-2-phthalimido- $\beta$-D-glucopyranosyl)-(1 $\rightarrow 6)-2,3,4$-tri$O$-benzyl-a-D-glucopyranoside (13)

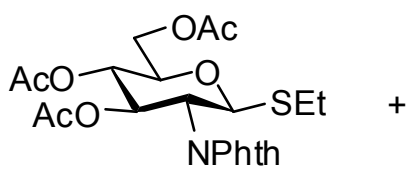

12

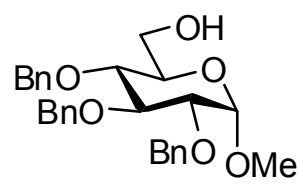

2

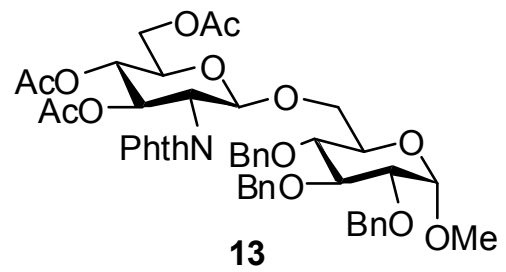

13

Reaction of $12^{12}$ with $2^{2}$ by procedure $a$ afforded 13 (85\%); mp 152-153 ${ }^{\circ} \mathrm{C}$ (from EtOAchexanes), lit. ${ }^{13} \mathrm{mp} 152-153{ }^{\circ} \mathrm{C} ;[\alpha]_{\mathrm{D}}+31\left(c 0.6, \mathrm{CHCl}_{3}\right)$, lit. $^{13}[\alpha]_{\mathrm{D}}+34\left(c 1.0, \mathrm{CHCl}_{3}\right)$, lit. ${ }^{14}$ $[\alpha]_{\mathrm{D}}+14.3\left(c 1.0, \mathrm{CHCl}_{3}\right)$, lit. ${ }^{15}[\alpha]_{\mathrm{D}}+42.5\left(c 1.0, \mathrm{CH}_{2} \mathrm{Cl}_{2}\right)$. The spectral data were consistent with those in the literature ${ }^{15}$.

Methyl [tert-butyl (2-O-benzoyl-3,4-di-O-benzyl- $\beta$-D-glucopyranosyl)uronate]-(1 $\rightarrow$ 4)-3$O$-benzyl-2-benzyloxycarbonylamino-2-deoxy-6-O-(4-methoxy)phenyl- $\alpha$-Dglucopyranoside (15)

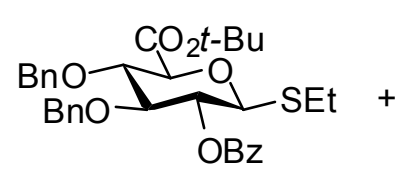

14

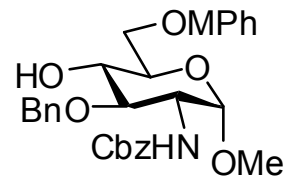

4

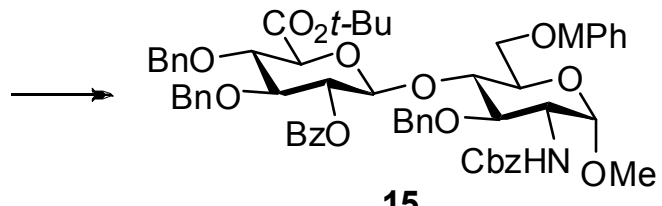

15

Reaction of 14 with $4^{5}$ by procedure $c$ afforded $15(83 \%)$; mp 128-129 ${ }^{\circ} \mathrm{C}$ (from ethyl acetatehexanes); $[\alpha]_{\mathrm{D}}+58\left(c 0.6, \mathrm{CHCl}_{3}\right) ;{ }^{1} \mathrm{H}$ NMR $\left(400 \mathrm{MHz}, \mathrm{CDCl}_{3}\right): \delta 7.91$ (d, 2H, aromatic), 7.54 (m, 1H, aromatic), 7.42-7.00 (m, 22H, aromatic), 6.84-6.76 (m, 4H, aromatic), 5.31 (dd, 1H, $J_{2}$, , $, 9.3 \mathrm{~Hz}, \mathrm{H}-2^{\prime}$ ) 5.02 (d, 1H, J $12.1 \mathrm{~Hz}, \mathrm{PhCH}_{2}$ ), 4.97 (s, 2H, $\mathrm{PhCH}_{2}$ ), 4.79 (d, 1H, $J_{1}^{\prime}, 2^{\prime}$ $7.8 \mathrm{~Hz}, \mathrm{H}-1$ '), 4.75 (d, 1H, J 10.8 Hz, $\mathrm{PhCH}_{2}$ ), 4.66 (d, 1H, J 10.8 Hz, $\mathrm{PhCH}_{2}$ ), 4.64 (d, 1H, J $\left.11.1 \mathrm{~Hz}, \mathrm{PhCH}_{2}\right), 4.64$ (d, 1H, J2,NH 9.1 Hz, NH), 4.63 (d, 1H, $\left.J_{1,2} 3.6 \mathrm{~Hz}, \mathrm{H}-1\right), 4.61$ (d, 1H, J $12.1 \mathrm{~Hz}, \mathrm{PhCH}_{2}$ ), 4.52 (d, 1H, J 11.1 Hz, $\mathrm{PhCH}_{2}$ ), 4.08 (dd, 1H, J,5 $\left.10.0 \mathrm{~Hz}, \mathrm{H}-4\right), 3.97$ (dd, 1H, $J_{4}, 5^{\prime}, 9.3 \mathrm{~Hz}, \mathrm{H}-4$ '), 3.96-3.93 (m, 3H, H-2, H-6a, H-6b), 3.80 (s, 3H, $\mathrm{ArOCH}_{3}$ ), 3.78 (d,

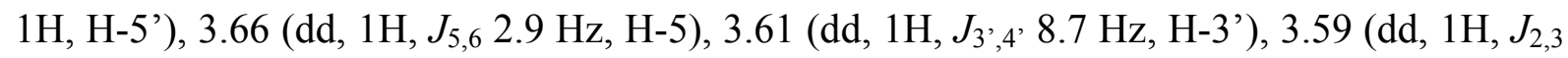
$\left.10.0 \mathrm{~Hz}, J_{3,4} 8.4 \mathrm{~Hz}, \mathrm{H}-3\right), 3.22\left(\mathrm{~s}, 3 \mathrm{H}, \mathrm{OCH}_{3}\right) ; 1.40\left(\mathrm{~s}, 9 \mathrm{H}, \mathrm{C}\left(\mathrm{CH}_{3}\right)_{3}\right) ;{ }^{13} \mathrm{C} \mathrm{NMR}(100 \mathrm{MHz}$, 


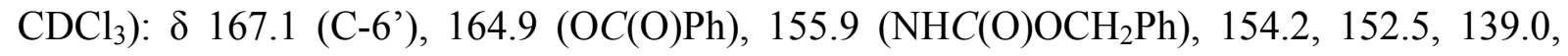
$137.8,137.5,136.4,133.3,129.8,129.5,128.5,128.4,128.2,128.1,127.9,127.8,127.74$, 127.66, 127.3, 115.8, 114.7 (aromatic), 101.0 (C-1'), $98.8(\mathrm{C}-1), 82.6\left(C\left(\mathrm{CH}_{3}\right)_{3}\right), 81.9$ (C-3'), 79.7 (C-4'), 78.6 (C-3), 77.8 (C-4), 75.8 (C-5'), $75.0\left(\mathrm{PhCH}_{2}\right), 74.9\left(\mathrm{PhCH}_{2}\right), 74.7\left(\mathrm{PhCH}_{2}\right)$, 73.9 (C-2'), $69.3(\mathrm{C}-5), 66.9\left(\mathrm{NHC}(\mathrm{O}) \mathrm{OCH} \mathrm{H}_{2} \mathrm{Ph}\right), 66.6(\mathrm{C}-6), 55.8\left(\mathrm{ArOCH}_{3}\right), 55.2\left(\mathrm{OCH}_{3}\right)$,

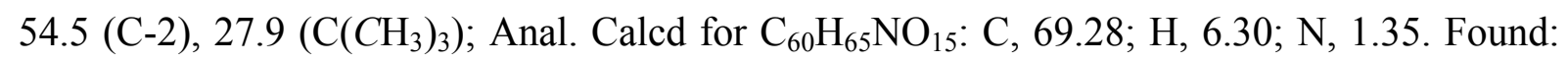
C, 69.31; H, 6.27; N, 1.34 .

Methyl (2,3,4,6-tetra- $O$-benzoyl- $\beta$-D-galactopyranosyl)-(1 $\rightarrow 6)-2,3,4$-tri- $O$-benzyl- $\alpha$-Dglucopyranoside (17)

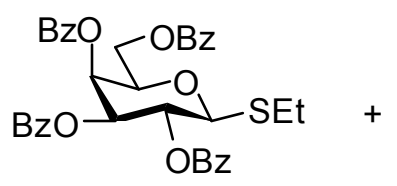

16

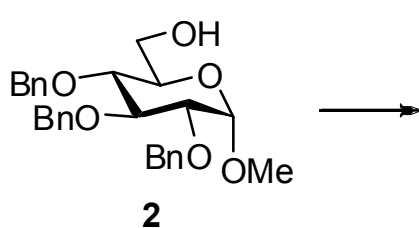

2

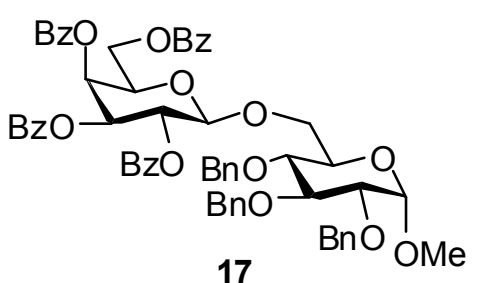

17

Reaction of $16^{16}$ with $2^{2}$ by procedure $a$ afforded $17(87 \%)$ as a syrup; $[\alpha]_{\mathrm{D}}+68(c 0.4$, $\mathrm{CHCl}_{3}$ ); ${ }^{1} \mathrm{H} \mathrm{NMR}\left(300 \mathrm{MHz}, \mathrm{CDCl}_{3}\right.$ ): $\delta 8.08$ (d, 2H, aromatic), 8.01 (d, 2H, aromatic), 7.98 (d, 2H, aromatic), 7.76 (d, 2H, aromatic), 7.64-7.10 (m, 27H aromatic), 5.97 (d, 1H, J4,, 2.9 Hz, H-4'), 5.85 (dd, 1H, $J_{2}^{\prime}, 3^{\prime} 10.3$ Hz, H-2'), 5.59 (dd, 1H, J', ${ }^{\prime}, 3.5$ Hz, H-3'), 4.90 (d, 1H, J $10.9 \mathrm{~Hz}, \mathrm{PhCH}_{2}$ ), 4.73 (d, 1H, $J_{1}, 2,8.1 \mathrm{~Hz}, \mathrm{H}-1$ '), 4.71 (d, 1H, J $12.2 \mathrm{~Hz}, \mathrm{PhCH}_{2}$ ), 4.69 (d, $\left.1 \mathrm{H}, J 10.6 \mathrm{~Hz}, \mathrm{PhCH}_{2}\right), 4.66$ (dd, $1 \mathrm{H}, J_{5^{\prime}, 6 \mathrm{a}}, 6.4 \mathrm{~Hz}, J_{6 \mathrm{a}}, 6 \mathrm{~b}, 10.9 \mathrm{~Hz}, \mathrm{H}-6 \mathrm{a}$ '), 4.58 (d, 1H, J 12.3 $\mathrm{Hz}, \mathrm{PhCH}_{2}$ ), 4.57 (d, 1H, J $11.1 \mathrm{~Hz}, \mathrm{PhCH}_{2}$ ), 4.50 (d, 1H, J,2 $3.4 \mathrm{~Hz}, \mathrm{H}-1$ ), 4.39 (dd, 1H, $\left.J_{5}{ }^{\prime}, 6 \mathrm{~b}, 6.8 \mathrm{~Hz}, \mathrm{H}-6 \mathrm{~b}^{\prime}\right), 4.38$ (d, 1H, J 11.1 Hz, $\mathrm{PhCH}_{2}$ ), 4.28-4.18 (m, 2H, H-6a, H-5'), 3.90 (t, 1H, $\left.J_{3,4} 9.2 \mathrm{~Hz}, \mathrm{H}-3\right), 3.79-3.72$ (m, 2H, H-5, H-6b), 3.39 (dd, 1H, J2,3 9.6 Hz, H-2), 3.37 (t, $\left.1 \mathrm{H}, J_{4,5} 9.2 \mathrm{~Hz}, \mathrm{H}-4\right), 3.21\left(\mathrm{~s}, 3 \mathrm{H}, \mathrm{OCH}_{3}\right) ;{ }^{13} \mathrm{C} \mathrm{NMR}\left(75 \mathrm{MHz}, \mathrm{CDCl}_{3}\right): \delta 166.0(\mathrm{OC}(\mathrm{O}) \mathrm{Ph})$, $165.57(\mathrm{OC}(\mathrm{O}) \mathrm{Ph}), 165.54(\mathrm{OC}(\mathrm{O}) \mathrm{Ph}), 165.1(\mathrm{OC}(\mathrm{O}) \mathrm{Ph}), 138.8,138.2,138.1,133.5,133.2$, $133.1,130.0,129.7,129.6,129.4,129.2$, 129.0, 128.7, 128.6, 128.42, 128.39, 128.35, 128.2, 128.0, 127.8, 127.6, 127.5, 127.4 (aromatic), 102.0 (C-1'), 97.8 (C-1), 81.9 (C-3), 79.8 (C-2), $77.4(\mathrm{C}-4), 75.5\left(\mathrm{PhCH}_{2}\right), 74.7\left(\mathrm{PhCH}_{2}\right), 73.3\left(\mathrm{PhCH}_{2}\right), 71.6(\mathrm{C}-3$ ') $71.3(\mathrm{C}-5$ ') 69.7 (C-2'), 69.6 (C-5), 68.7 (C-6), 68.1 (C-4'), 61.9 (C-6'), $55.0\left(\mathrm{OCH}_{3}\right)$; Anal. Calcd for $\mathrm{C}_{62} \mathrm{H}_{58} \mathrm{O}_{15}$ : C, 71.39; H, 5.60. Found: C, 71.14; H, 5.63. 
Methyl (2,3,4,6-tetra-O-benzoyl- $\beta$-D-galactopyranosyl)-(1 $\rightarrow 4)-2,3-$ di- $O$-benzoyl-6- $O$ benzyl- $\alpha$-D-glucopyranoside (19)

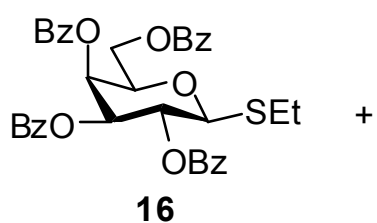

16

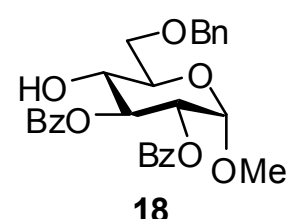

18

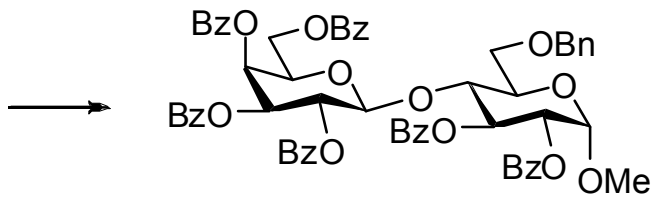

19

Reaction of $16^{16}$ with $18^{6}$ by procedure $a$ afforded 19 (85\%); mp 103-104 ${ }^{\circ} \mathrm{C}$ (from ethyl acetate-hexanes); $[\alpha]_{\mathrm{D}}+30\left(c 0.6, \mathrm{CHCl}_{3}\right) ;{ }^{1} \mathrm{H} \mathrm{NMR}\left(300 \mathrm{MHz}, \mathrm{CDCl}_{3}\right): \delta 8.06-7.95(\mathrm{~m}, 6 \mathrm{H}$, aromatic), 7.89-7.79 (m, $4 \mathrm{H}$, aromatic), $7.73(\mathrm{~d}, 2 \mathrm{H}$, aromatic), 7.63-7.14 (m, 23H, aromatic), 5.97 (t, 1H, J3,4 $9.8 \mathrm{~Hz}, \mathrm{H}-3), 5.71$ (d, 1H, $J_{4}, 5,3.0 \mathrm{~Hz}, \mathrm{H}-4$ '), 5.59 (dd, 1H, $J_{2}{ }^{3}, 10.3 \mathrm{~Hz}, \mathrm{H}-$ 2'), 5.25 (dd, 1H, J', $J^{\prime}, 3.3 \mathrm{~Hz}, \mathrm{H}-3$ '), 5.21 (dd, 1H, J $\left.{ }_{2,3} 9.5 \mathrm{~Hz}, \mathrm{H}-2\right), 5.16$ (d, 1H, J1,2 $3.6 \mathrm{~Hz}$, H-1), 4.83 (d, 1H, J 12.1 Hz, $\mathrm{PhCH}_{2}$ ), 4.69 (d, 1H, $J_{1}, 2,8.0$ Hz, H-1'), 4.42 (d, 1H, J $12.1 \mathrm{~Hz}$, $\mathrm{PhCH}_{2}$ ), 4.30 (t, 1H, J4,5 9.7 Hz, H-4), 3.90-3.71 (m, 5H, H-5, H-6a, H-6b, H-5', H-6a',), 3.55 (d, 1H, $J_{6 \mathrm{a}, 6 \mathrm{~b}} 10.0 \mathrm{~Hz}, \mathrm{H}-6 \mathrm{~b}$ '), $3.33\left(\mathrm{~s}, 3 \mathrm{H}, \mathrm{OCH}_{3}\right) ;{ }^{13} \mathrm{C} \mathrm{NMR}\left(75 \mathrm{MHz}, \mathrm{CDCl}_{3}\right): \delta 165.9$ $(\mathrm{OC}(\mathrm{O}) \mathrm{Ph}), 165.7(\mathrm{OC}(\mathrm{O}) \mathrm{Ph}), 165.4(2 \mathrm{x} \mathrm{OC}(\mathrm{O}) \mathrm{Ph}), 165.3(\mathrm{OC}(\mathrm{O}) \mathrm{Ph}), 164.6(\mathrm{OC}(\mathrm{O}) \mathrm{Ph})$, $137.8,133.3,133.2,132.8,130.1,129.9,129.7,129.5,129.2,128.8,128.7,128.6,128.5$, 128.41, 128.35, 128.3, 128.2, 128.1 (aromatic), 100.2 (C-1'), 97.1 (C-1), 74.6 (C-4), 73.7 $\left(\mathrm{PhCH}_{2}\right), 72.0$ (C-2), 71.8 (C-3'), 71.0 (C-5'), 70.4 (C-3), 70.0 (C-2'), 69.6 (C-5), 67.6 (C-4'), 67.1 (C-6), $61.3\left(\mathrm{C}-6\right.$ '), $55.5\left(\mathrm{OCH}_{3}\right)$; Anal. Calcd for $\mathrm{C}_{62} \mathrm{H}_{54} \mathrm{O}_{17}$ : C, 69.52; H, 5.08. Found: C, 69.26; H, 5.06 .

\section{Methyl (2,3,4,6-tetra- $O$-benzoyl- $\beta$-D-galactopyranosyl)-(1 $\rightarrow 4)-3-O$-benzyl-2-}

benzyloxycarbonylamino-2-deoxy-6-O-(4-methoxy)phenyl- $\alpha$-D-glucopyranoside (20)
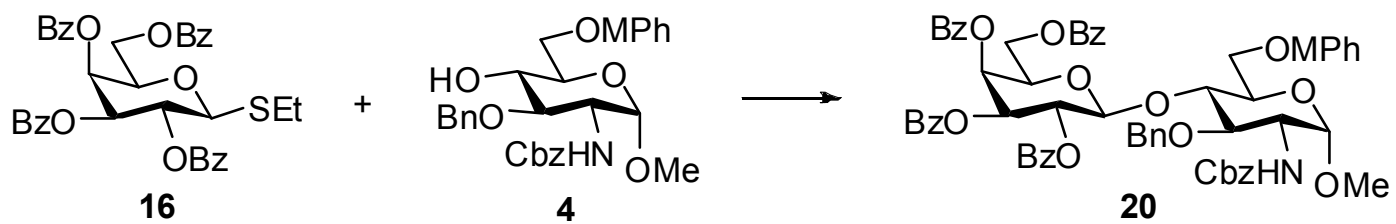

Reaction of $16^{16}$ with $4^{5}$ by procedure $a$ afforded $20(79 \%)$ as a white foam; $[\alpha]_{\mathrm{D}}+84(c 0.5$, $\mathrm{CHCl}_{3}$ ); ${ }^{1} \mathrm{H} \mathrm{NMR}\left(300 \mathrm{MHz}, \mathrm{CDCl}_{3}\right.$ ): $\delta$ 8.03-7.96 (m, 4H, aromatic), 7.90 (d, 2H, aromatic), $7.73(\mathrm{~d}, 2 \mathrm{H}$, aromatic), 7.60-7.16 (m, 22H, aromatic), 6.87-6.75 (m, 4H, aromatic), 5.87 (d, $1 \mathrm{H}, J_{4^{\prime}, 5}, 3.3 \mathrm{~Hz}, \mathrm{H}-4^{\prime}$ ), 5.80 (dd, 1H, $J_{2}, 3^{\prime}, 10.3 \mathrm{~Hz}, \mathrm{H}-2^{\prime}$ ), 5.37 (dd, 1H, $J_{3}, 4,3.3 \mathrm{~Hz}, \mathrm{H}-3$ '), 5.14 (d, 1H, J $12.5 \mathrm{~Hz}, \mathrm{PhCH}_{2}$ ), 5.13 (d, 1H, J $12.5 \mathrm{~Hz}, \mathrm{PhCH}_{2}$ ), 5.03 (d, 1H, J $12.5 \mathrm{~Hz}$, $\mathrm{PhCH}_{2}$ ), 4.95 (d, 1H, $\left.J_{1}{ }^{2}, 2.1 \mathrm{~Hz}, \mathrm{H}-1^{\prime}\right), 4.82$ (d, 1H, J $11.7 \mathrm{~Hz}, \mathrm{PhCH}_{2}$ ), 4.81 (d, 1H, J 9.2 Hz, NH), 4.70 (d, 1H, $J_{1,2} 2.9$ Hz, H-1), 4.35 (dd, 1H, $\left.J_{5}{ }^{\prime}, 6 \mathrm{a}^{\prime} 6.2 \mathrm{~Hz}, J_{6 \mathrm{a}^{\prime}, 6 \mathrm{~b}^{\prime}} 11.4 \mathrm{~Hz}, \mathrm{H}-6 \mathrm{a}^{\prime}\right)$, 
4.22 (t, 1H, $\left.J_{4,5} 9.6 \mathrm{~Hz}, \mathrm{H}-4\right), 4.22$ (dd, 1H, $J_{5}$, 6b' 3.4 Hz, H-6b') 4.09-3.96 (m, 4H, H-2, H-6a, H-6b, H-5'), 3.81 (s, 3H, $\mathrm{ArOCH}_{3}$ ), 3.76 (m, 1H, H-5), 3.70 (t, 1H, $J_{2,3}=J_{3,4} 9.6$ Hz, H-3), 3.27 (s, 3H, $\left.\mathrm{OCH}_{3}\right) ;{ }^{13} \mathrm{C} \mathrm{NMR}\left(75 \mathrm{MHz}, \mathrm{CDCl}_{3}\right): \delta 165.9(\mathrm{OC}(\mathrm{O}) \mathrm{Ph}), 165.43(\mathrm{OC}(\mathrm{O}) \mathrm{Ph}), 165.42$ $(\mathrm{OC}(\mathrm{O}) \mathrm{Ph}), 165.0(\mathrm{OC}(\mathrm{O}) \mathrm{Ph}), 155.9\left(\mathrm{NHC}(\mathrm{O}) \mathrm{OCH}_{2} \mathrm{Ph}\right), 154.3,152.3,138.7,136.3,133.5$, 133.2 , 129.8, 129.7, 129.4, 129.1, 128.9, 128.8, 128.7, 128.6, 128.5, 128.2, 127.5, 127.4, 115.6, 114.8 (aromatic), 100.8 (C-1'), 98.9 (C-1), 78.3 (C-3), 77.4 (C-4), 74.4 ( $\left.\mathrm{PhCH}_{2}\right), 71.9$ (C-3'), 71.2 (C-5'), 70.5 (C-2'), 69.4 (C-5), 67.8 (C-4'), 66.9 (NHC(O)OCH $\left.{ }_{2} \mathrm{Ph}\right), 66.4$ (C-6'), $61.4(\mathrm{C}-6), 55.7\left(\mathrm{ArOCH}_{3}\right), 55.3\left(\mathrm{OCH}_{3}\right), 54.5(\mathrm{C}-2)$; Anal. Calcd for $\mathrm{C}_{63} \mathrm{H}_{59} \mathrm{NO}_{17}$ : C, 68.66; H, 5.40; N, 1.27. Found: C, 68.42; H, 5.41; N, 1.25.

Methyl [2-O-benzoyl-3-O-benzyl-4,6-O-(1-naphthyl)methylidene- $\beta$-D-glucopyranosyl]$(1 \rightarrow 4)-3-O$-benzyl-2-benzyloxycarbonylamino-2-deoxy-6-O-(4-methoxy)phenyl- $\alpha$-Dglucopyranoside (22)

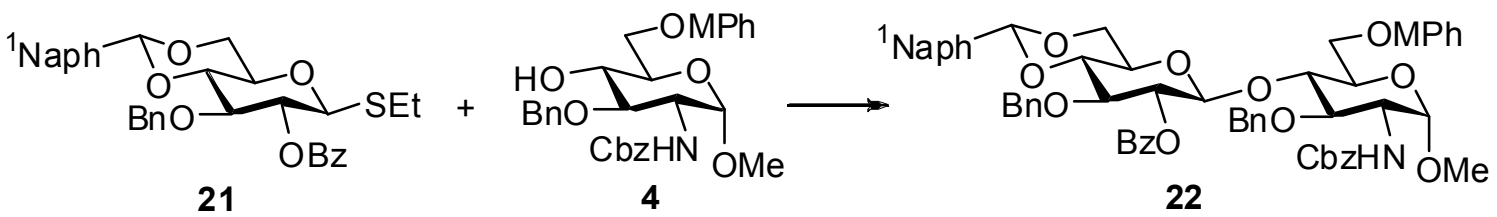

Reaction of 21 with $4^{5}$ by procedure $c$ afforded 22 (88\%); mp 198-199 ${ }^{\circ} \mathrm{C}$ (from ethyl acetatehexanes); $[\alpha]_{\mathrm{D}}+91\left(c \quad 0.5, \mathrm{CHCl}_{3}\right) ;{ }^{1} \mathrm{H} \mathrm{NMR}\left(300 \mathrm{MHz}, \mathrm{CDCl}_{3}\right): \delta 8.09$ (m, 1H, aromatic), 7.95-7.84 (m, 4H, aromatic), $7.76(\mathrm{~d}, 1 \mathrm{H}$, aromatic), $7.60(\mathrm{t}, 1 \mathrm{H}$, aromatic), 7.52-7.23 (m, $15 \mathrm{H}$, aromatic), $7.06(\mathrm{~m}, 1 \mathrm{H}$, aromatic), 7.00-6.88 (m, 4H, aromatic), 6.85-6.73 (m, 4H, aromatic), 6.01 (s, 1H, $\left.{ }^{1} \mathrm{NaphCH}\right), 5.33$ (t, 1H, $J_{2}, 3,8.4 \mathrm{~Hz}, \mathrm{H}-2$ '), 5.10 (d, 1H, J $12.2 \mathrm{~Hz}$, $\mathrm{PhCH}_{2}$ ), 5.02 (d, 1H, J $\left.12.6 \mathrm{~Hz}, \mathrm{PhCH}_{2}\right), 4.96$ (d, 1H, J $\left.11.7 \mathrm{~Hz}, \mathrm{PhCH}_{2}\right), 4.81$ (d, 1H, J2,NH $9.7 \mathrm{~Hz}, \mathrm{NH}), 4.75$ (d, 1H, $J_{1^{\prime}, 2^{2}} 8.0 \mathrm{~Hz}, \mathrm{H}-1^{\prime}$ ), 4.68 (d, 1H, $\left.J_{1,2} 4.3 \mathrm{~Hz}, \mathrm{H}-1\right), 4.67$ (d, 1H, J $11.1 \mathrm{~Hz}, \mathrm{PhCH}_{2}$ ), 4.66 (d, 1H, J 11.4 Hz, $\mathrm{PhCH}_{2}$ ), 4.43 (d, 1H, J 11.8 Hz, $\mathrm{PhCH}_{2}$ ), 4.26 (dd,

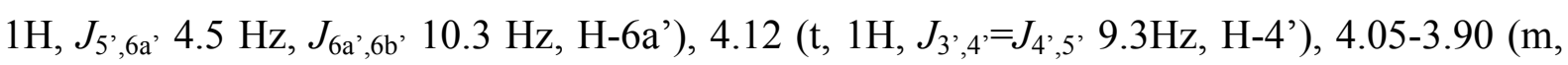
$3 \mathrm{H}, \mathrm{H}-2, \mathrm{H}-6 \mathrm{a}, \mathrm{H}-6 \mathrm{~b}$ ), 3.88 (t, $1 \mathrm{H}, J_{2,3}=J_{3,4} 9.2 \mathrm{~Hz}, \mathrm{H}-3$ ), 3.79 (s, 3H, $\mathrm{ArOCH}_{3}$ ), 3.71-3.40 (m,

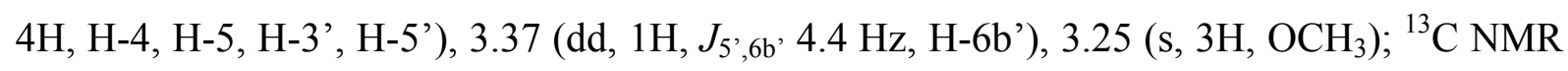
(75 MHz, $\left.\left.\mathrm{CDCl}_{3}\right): \delta 164.9(\mathrm{OC}(\mathrm{O}) \mathrm{Ph}), 155.9\left(\mathrm{NHC}^{\mathrm{O}}\right) \mathrm{OCH}_{2} \mathrm{Ph}\right), 154.2,152.4,139.0,137.5$, $136.3,133.7,133.3,132.2,130.4,129.9,129.7,129.4,128.6,128.4,128.2,128.0,127.9$, 127.4, 127.3, 126.3, 125.7, 124.9, 124.0, 123.8, 115.7, 114.6 (aromatic), 101.3 (C-1'), 100.2 ( $\left.{ }^{1} \mathrm{NaphCH}\right), 98.9$ (C-1), 82.1 (C-3), 78.6 (C-3'), 78.2 (C-4), 77.5 (C-4'), $74.8\left(\mathrm{PhCH}_{2}\right), 74.1$ $\left(\mathrm{PhCH}_{2}\right), 74.0$ (C-2'), 69.4 (C-5), 68.7 (C-6'), 66.9 (NHC(O)OCH$\left.{ }_{2} \mathrm{Ph}\right), 66.2(\mathrm{C}-6), 66.1$ (C- 
5'), $55.7\left(\mathrm{ArOCH}_{3}\right), 55.2\left(\mathrm{OCH}_{3}\right), 54.5(\mathrm{C}-2)$; Anal. Calcd for $\mathrm{C}_{60} \mathrm{H}_{59} \mathrm{NO}_{14}$ : C, 70.78; H, 5.84; N, 1.38. Found: C, 70.60; H, 5.86; N, 1.37.

Methyl [2-O-benzoyl-3-O-benzyl-4,6-O-(1-naphthyl)methylidene- $\alpha$-L-idopyranosyl]$(1 \rightarrow 4)-3-O$-benzyl-2-benzyloxycarbonylamino-2-deoxy-6-O-(4-methoxy)phenyl- $\alpha$-Dglucopyranoside (24)

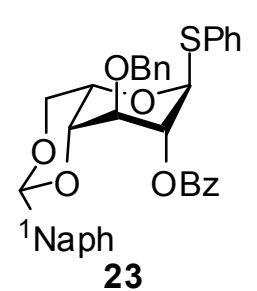

23
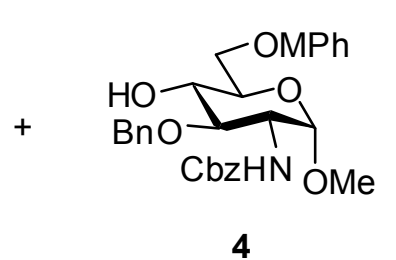

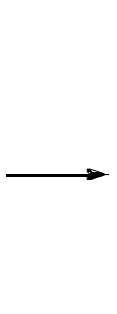

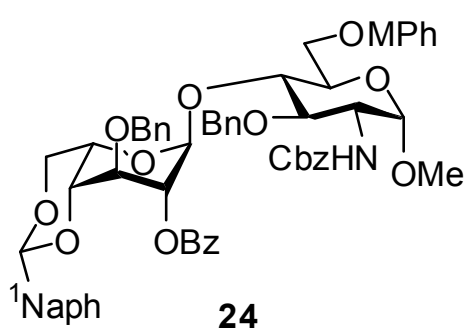

24

Reaction of $23^{17}$ with $4^{5}$ by procedure $c$ afforded 24 (90\%); mp 209-210 ${ }^{\circ} \mathrm{C}$ (from ethyl acetate-hexanes); $[\alpha]_{\mathrm{D}}-17\left(c\right.$ 0.8, $\left.\mathrm{CHCl}_{3}\right) ;{ }^{1} \mathrm{H}$ NMR (300 MHz, $\left.\mathrm{CDCl}_{3}\right): \delta 8.42-6.60(\mathrm{~m}, 31 \mathrm{H}$, aromatic), 5.80 (s, 1H, $\left.{ }^{1} \mathrm{NaphCH}\right), 5.24$ (d, 1H, $J_{2}, 3^{\prime}, 1.5 \mathrm{~Hz}, \mathrm{H}-2$ '), 5.13 (s, 1H, H-1'), $5.07-$ 4.98 (m, 3H, $\left.\mathrm{PhCH}_{2}, \mathrm{NH}\right), 4.81$ (d, 1H, J $\left.11.7 \mathrm{~Hz}, \mathrm{PhCH}_{2}\right), 4.77$ (d, 1H, J1,2 $\left.4.4 \mathrm{~Hz}, \mathrm{H}-1\right)$, 4.66 (s, 2H, $\left.\mathrm{PhCH}_{2}\right), 4.65$ (d, 1H, J $11.7 \mathrm{~Hz}, \mathrm{PhCH}_{2}$ ), 4.30-4.14 (m, 4H, H-2, H-4, H-6a, H6b), 4.06 (s, 1H, H-5'), 4.00-3.90 (m, 3H, H-5, H-4', H-6a'), 3.76 (t, 1H, J', , 2.2 Hz, H-3'), $3.63\left(\mathrm{t}, 1 \mathrm{H}, J_{3,4} 9.5 \mathrm{~Hz}, \mathrm{H}-3\right), 3.60\left(\mathrm{~s}, 3 \mathrm{H}, \mathrm{ArOCH}_{3}\right), 3.42$ (s, 3H, OCH 3$), 3.19$ (d, 1H, J6a',6b' $12.4 \mathrm{~Hz}, \mathrm{H}-6 \mathrm{~b}) ;{ }^{13} \mathrm{C}$ NMR (75 MHz, $\left.\mathrm{CDCl}_{3}\right): \delta 165.8(\mathrm{OC}(\mathrm{O}) \mathrm{Ph}), 155.9\left(\mathrm{NHC}(\mathrm{O}) \mathrm{OCH} \mathrm{H}_{2} \mathrm{Ph}\right)$, $154.0,152.2,138.4,137.8,136.3,133.9,133.5,132.9,130.6,130.0,129.7,129.1,128.5$, $128.4,128.3,128.1,127.99,127.95,127.9,127.4,127.3,126.3,125.6,125.3,125.1,124.7$, 115.8, 114.5 (aromatic), $101.4\left({ }^{1} \mathrm{NaphCH}\right), 99.1$ (C-1'), 98.0 (C-1), 79.7 (C-3), 75.0 (C-3'), $74.9\left(\mathrm{PhCH}_{2}\right), 73.8,73.6$ (C-4, C-5), $72.2\left(\mathrm{PhCH}_{2}\right), 70.4$ (C-4'), $69.3(\mathrm{C}-6$ ') , 67.0, 66.9, 66.8 (C-2', C-6, $\left.\mathrm{NHC}(\mathrm{O}) \mathrm{OCH} \mathrm{H}_{2} \mathrm{Ph}\right), 59.8$ (C-5'), 55.5, $55.4\left(\mathrm{ArOCH}_{3}, \mathrm{OCH}_{3}\right), 55.3$ (C-2); Anal. Calcd for $\mathrm{C}_{60} \mathrm{H}_{59} \mathrm{NO}_{14}$ : C, 70.78; H, 5.84; N, 1.38. Found: C, 70.53; H, 5.87; N, 1.39.

Methyl (2,3,4,6-tetra- $O$-acetyl- $\alpha$-D-mannopyranosyl)-(1 $\rightarrow 4)-2,3,6$-tri- $O$-benzyl- $\alpha$-Dglucopyranoside (26)

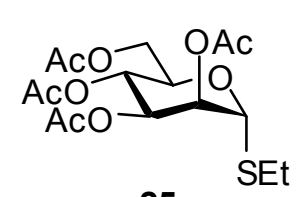

25

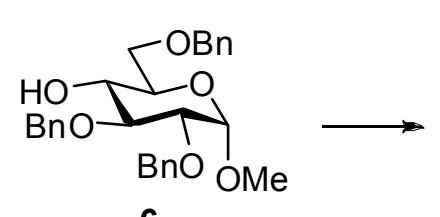

6

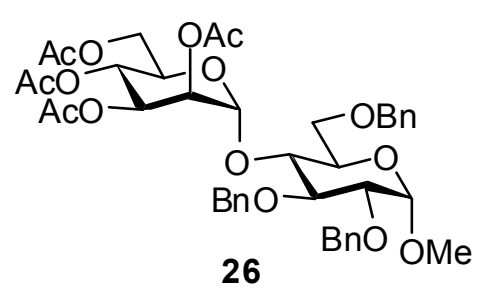

26 
Reaction of $25^{18}$ with $6^{6}$ by procedure $a$ afforded $26(79 \%)$ as a syrup; $[\alpha]_{\mathrm{D}}+41(c 0.4$, $\left.\mathrm{CHCl}_{3}\right)$, lit. ${ }^{19}[\alpha]_{\mathrm{D}}+43\left(c 1.0, \mathrm{CHCl}_{3}\right)$. The spectral data were consistent with those in the literature $^{19}$.

Methyl (2,3,4,6-tetra- $O$-benzyl- $\alpha$-D-glucopyranosyl)-(1 $\rightarrow 4)-2,3,6$-tri- $O$-benzyl- $\alpha$-Dglucopyranoside $(28)$ and methyl $(2,3,4,6$-tetra- $O$-benzyl- $\beta$-D-glucopyranosyl)-( $1 \rightarrow 4)$ 2,3,6-tri-O-benzyl- $\alpha$-D-glucopyranoside (29)

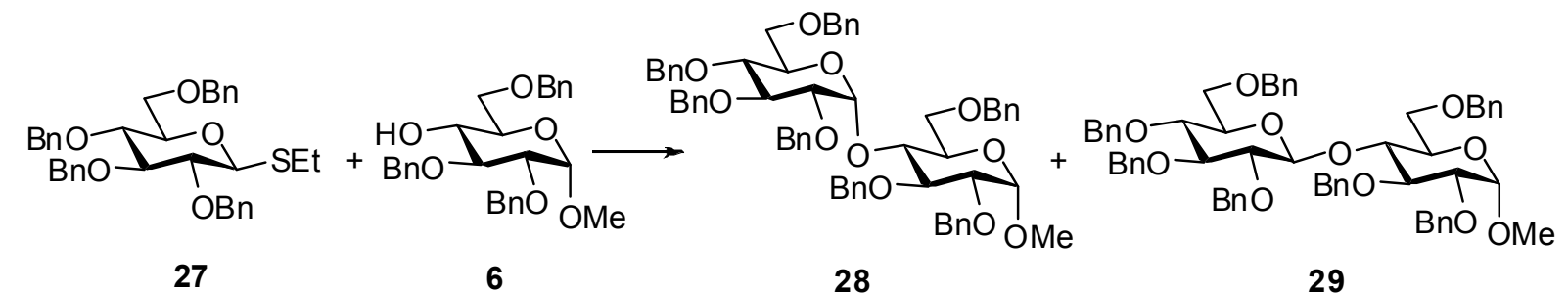

Reaction of $27^{20}$ with $\mathbf{6}^{6}$ by procedure $c$ afforded 28 and 29 in $44 \%$ and $43 \%$ yields, respectively. By using procedure $d$ the corresponding yields were $70 \%$ and $23 \%$, whereas procedure $e$ afforded 28 and 29 in $23 \%$ and $63 \%$ yields. 28: syrup, $[\alpha]_{\mathrm{D}}+46\left(c 0.6, \mathrm{CHCl}_{3}\right)$, lit. $^{3}[\alpha]_{\mathrm{D}}+40\left(c 1.1, \mathrm{CHCl}_{3}\right)$, lit. $^{21}[\alpha]_{\mathrm{D}}+47\left(c 0.87, \mathrm{CHCl}_{3}\right)$. The spectral data were consistent with those in the literature ${ }^{3,21}$. 29: $\mathrm{mp} 79-81{ }^{\circ} \mathrm{C}$ (from ether-hexanes), lit. ${ }^{21} \mathrm{mp} \mathrm{83-85}{ }^{\circ} \mathrm{C}$, lit. ${ }^{3}$ mp 88-89 ${ }^{\circ} \mathrm{C} ;[\alpha]_{\mathrm{D}}+22\left(c 0.4, \mathrm{CHCl}_{3}\right)$, lit. $^{21}[\alpha]_{\mathrm{D}}+21\left(c 1.0, \mathrm{CHCl}_{3}\right)$, lit. $^{3}[\alpha]_{\mathrm{D}}+20(c 1.0$, $\mathrm{CHCl}_{3}$ ). The spectral data were consistent with those in the literature ${ }^{3,21}$.

\section{References}

1. Fügedi, P. ; Garegg, P. J. Carbohydr. Res. 1986, 149, C9-C12.

2. Küster, J. M.; Dyong, I. Liebigs Ann. Chem. 1975, 2179-2189.

3. Garcia, B. A.; Gin, D. Y. J. Am. Chem. Soc. 2000, 122, 4269-4279.

4. Kim, W.-S.; Hosono, S.; Sasai, H.; Shibasaki, M. Heterocycles 1996, 42, 795-809.

5. Petitou, M.; Duchuassoy, P.; Choay, J. Tetrahedron Lett. 1988, 29, 1389-1390.

6. Garegg, P. J. ; Hultberg, H. Carbohydr. Res. 1981, 93, C10-C11.

7. Marra, A.; Esnault, J.; Veyrières, A.; Sinaÿ, P. J. Am. Chem. Soc. 1992, 114, 6354-6360. 
8. Garegg, P. J. ; Iversen, T.; Oscarson, S. Carbohydr. Res. 1976, 50, C12-C14.

9. Ekelöf, K.; Oscarson, S. Carbohydr. Res. 1995, 278, 289-300.

10. Dasgupta, F.; Garegg, P. J. Carbohydr. Res. 1988, 177, C13-C17.

11. Dondoni, A.; Marra, A.; Massi, A. Angew. Chem., Int. Ed. 2005, 44, 1672-1676.

12. Lönn, H. Carbohydr. Res. 1985, 139, 105-113.

13. Balavoine, G.; Berteina, S.; Gref, A.; Fischer, J.-C.; Lubineau, A. J. Carbohydr. Chem. 1995, 14, 1237-1249.

14. Bongat, A. F. G.; Kamat, M. N.; Demchenko, A. V. J. Org. Chem. 2007, 72, 1480-1483.

15. Mehta, S.; Pinto, B. M. J. Org. Chem. 1993, 58, 3269-3276.

16. Cao, S.; Hernández-Mateo, F.; Roy, R. J. Carbohydr. Chem. 1998, 17, 609-631.

17. Tatai, J.; Osztrovszky, G.; Kajtár-Peredy, M.; Fügedi, P. Carbohydr. Res. submitted.

18. Lemieux, R. U.; Brice, C. Can. J. Chem. 1955, 33, 109-119.

19. Mukhopadhyay, B.; Maurer, S. V.; Rudolph, N.; van Well, R. M.; Russell, D. A.; Field, R. A. J. Org. Chem. 2005, 70, 9059-9062.

20. Weygand, F.; Ziemann, H. Liebigs Ann. Chem. 1962, 657, 179-198.

21. Jona, H.; Mandai, H.; Chavasiri, W.; Takeuchi, K.; Mukaiyama, T. Bull. Chem. Soc. Jpn. 2002, 75, 291-309. 


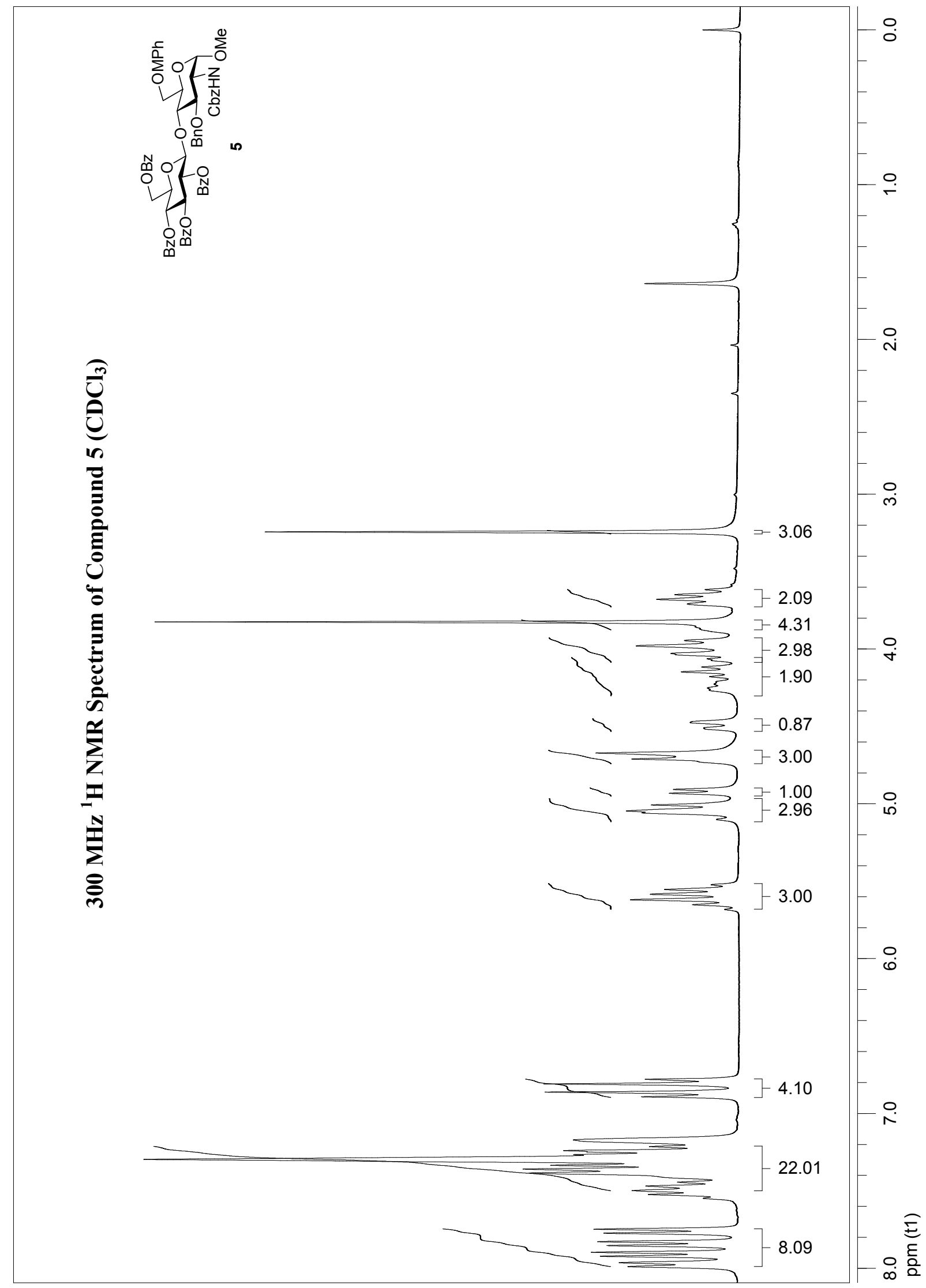




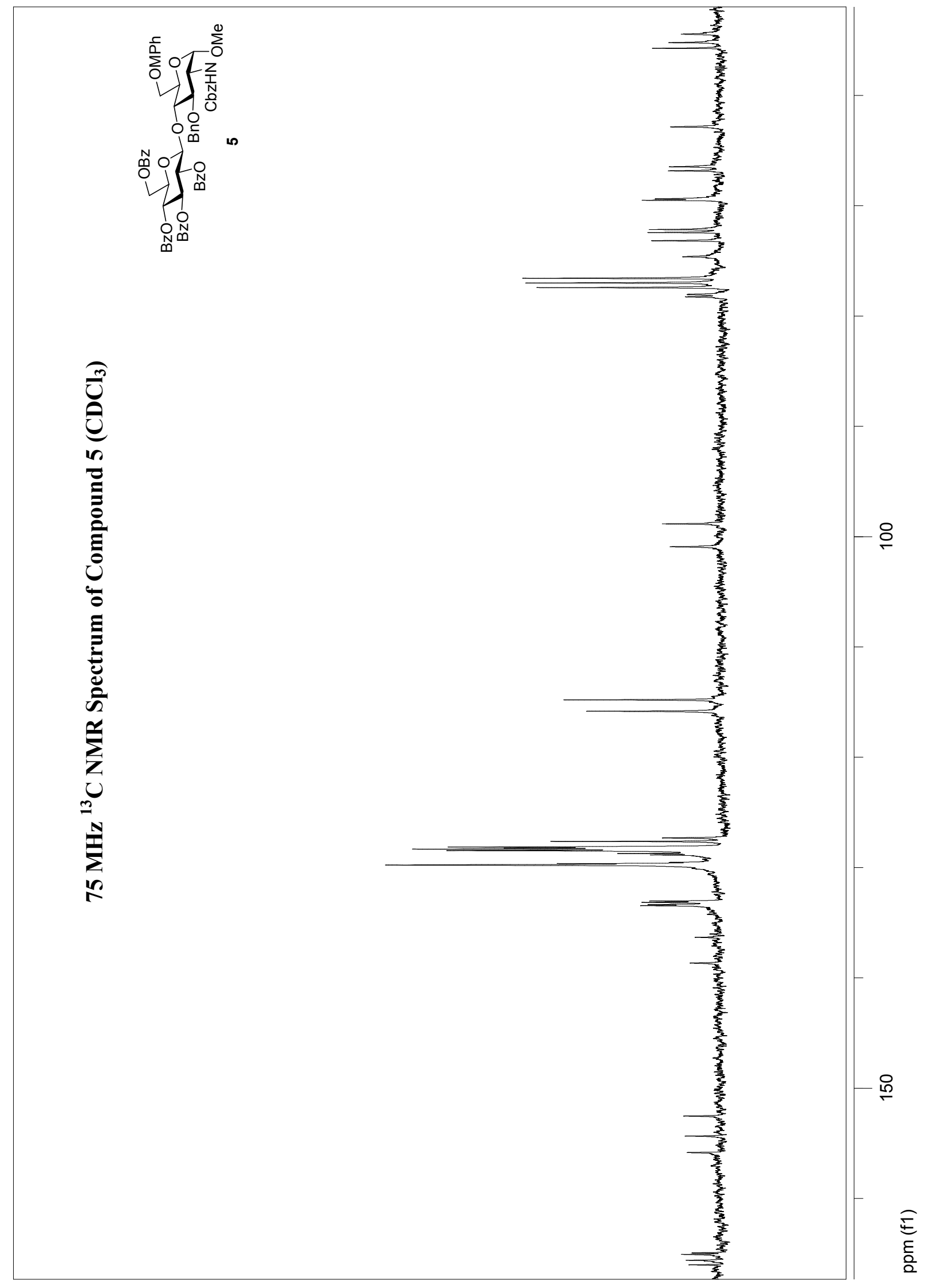


$300 \mathrm{MHz}$ COSY Spectrum of Compound $5\left(\mathrm{CDCl}_{3}\right)$
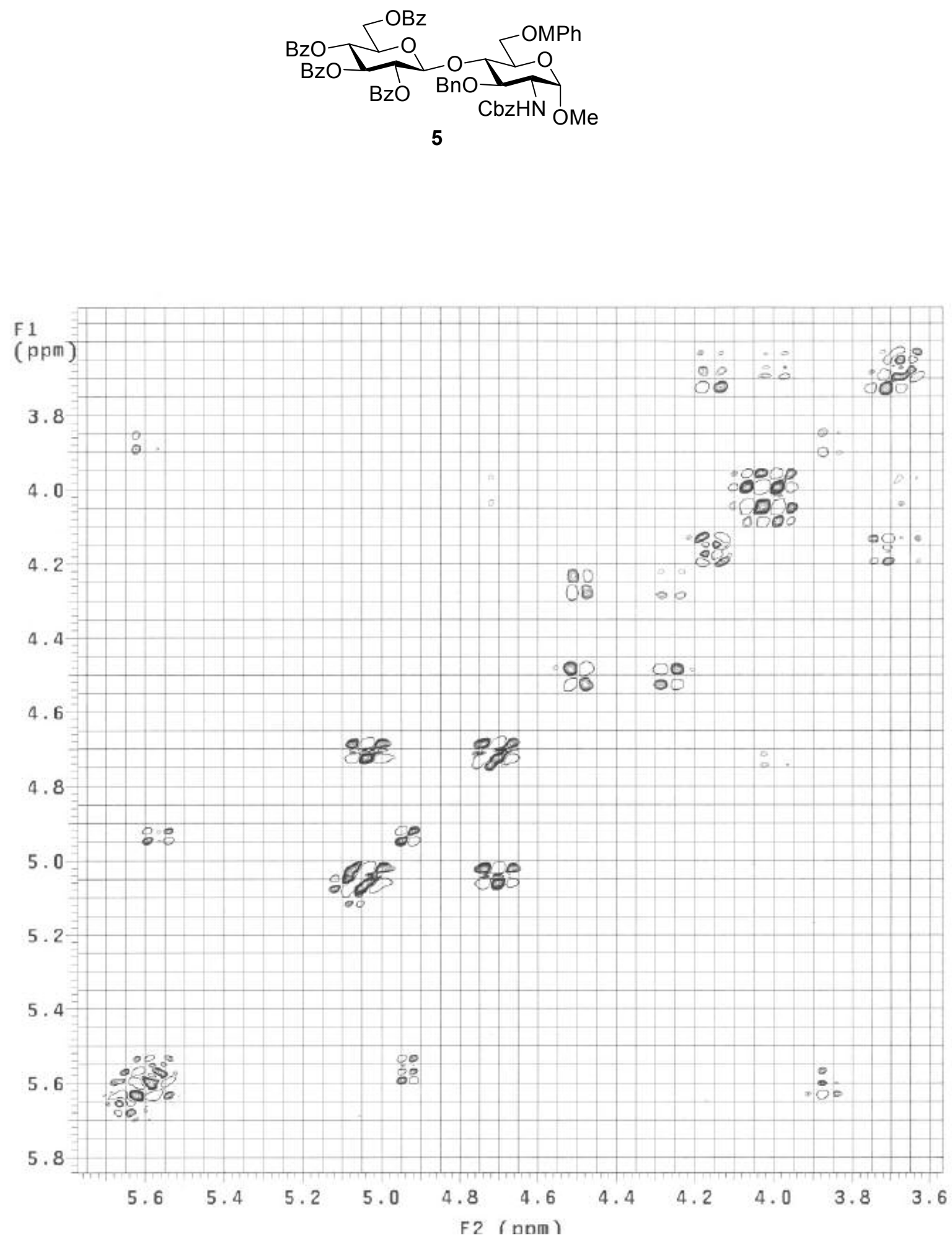
HSQC Spectrum of Compound $5\left(\mathrm{CDCl}_{3}\right)$
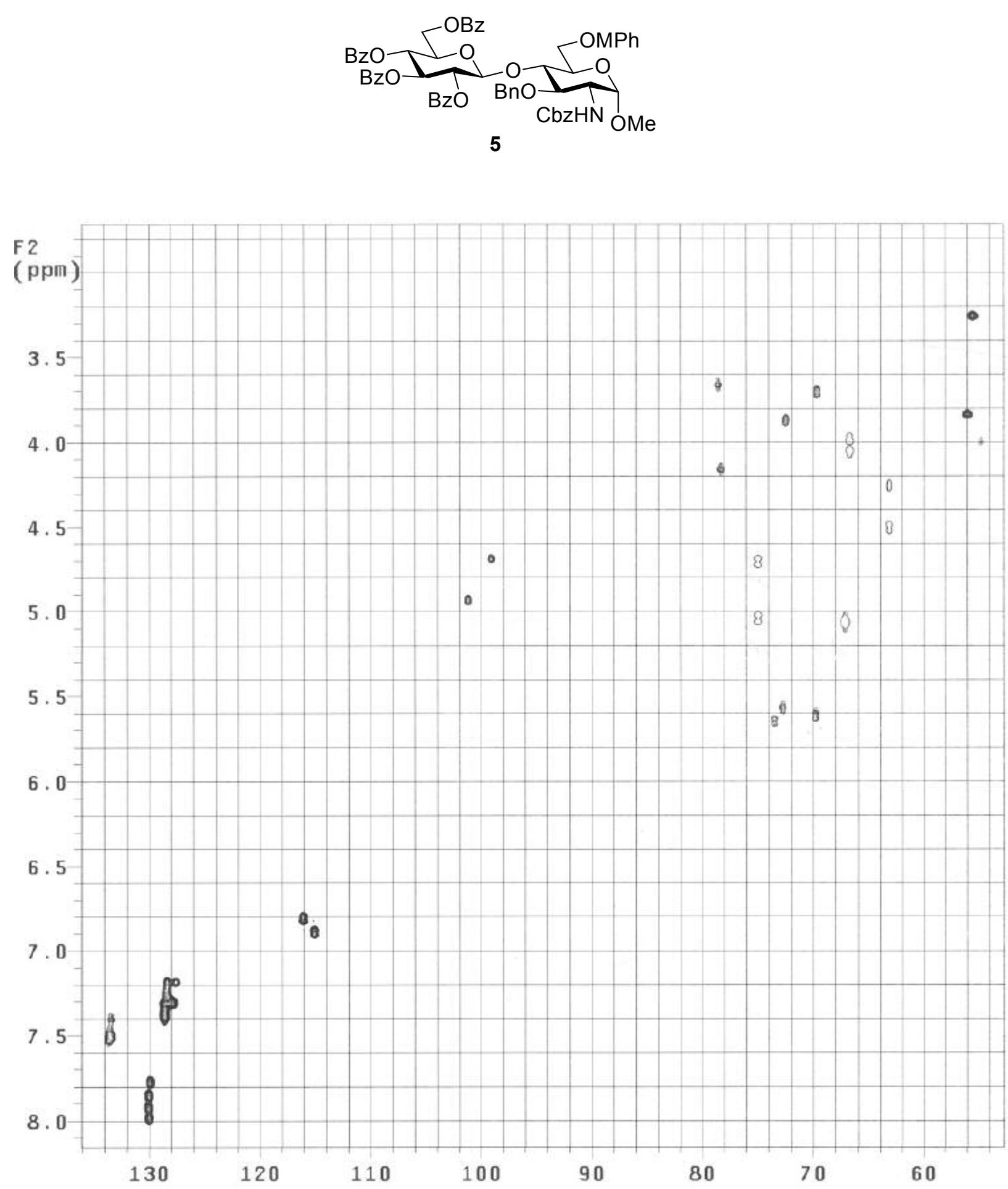


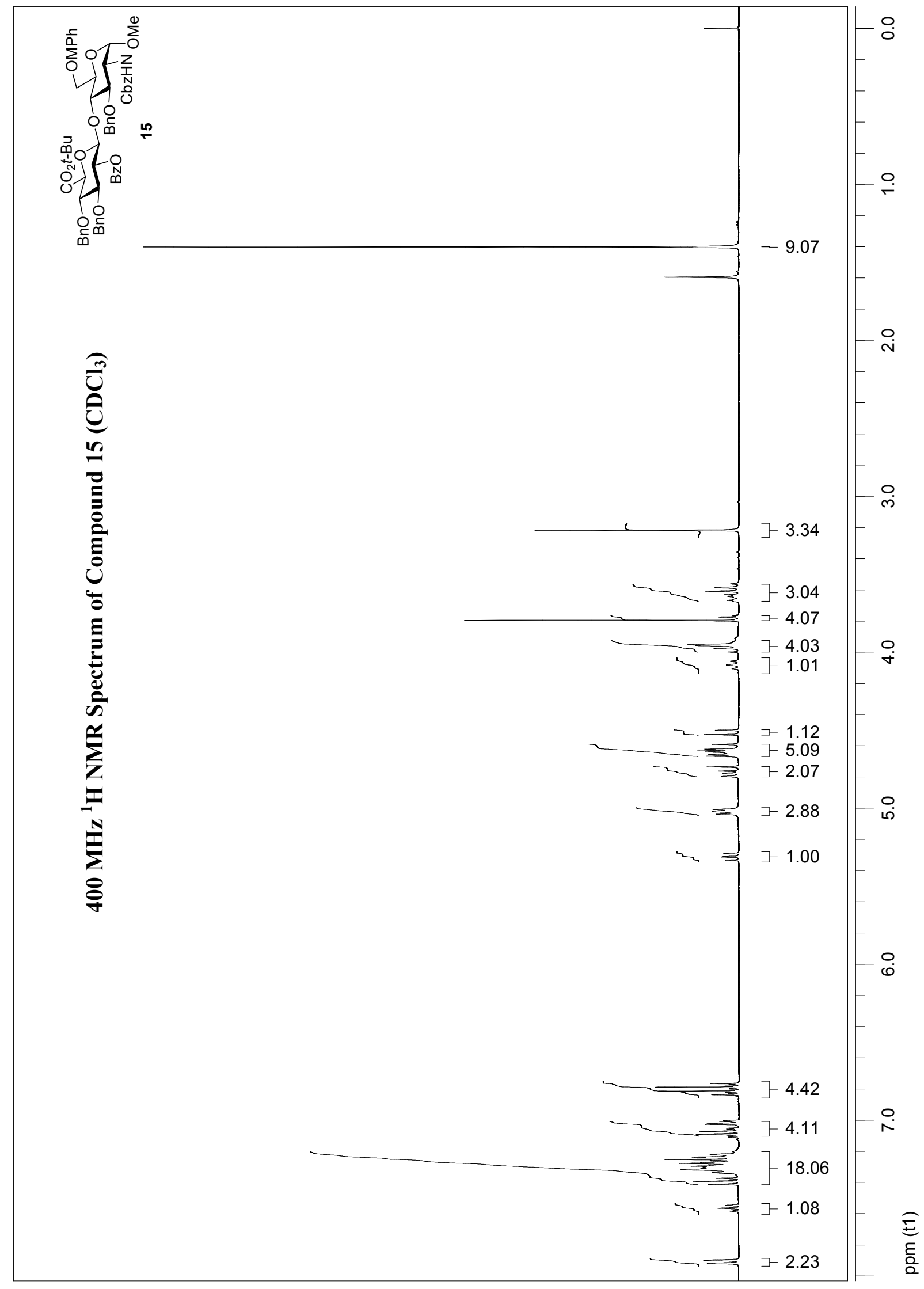




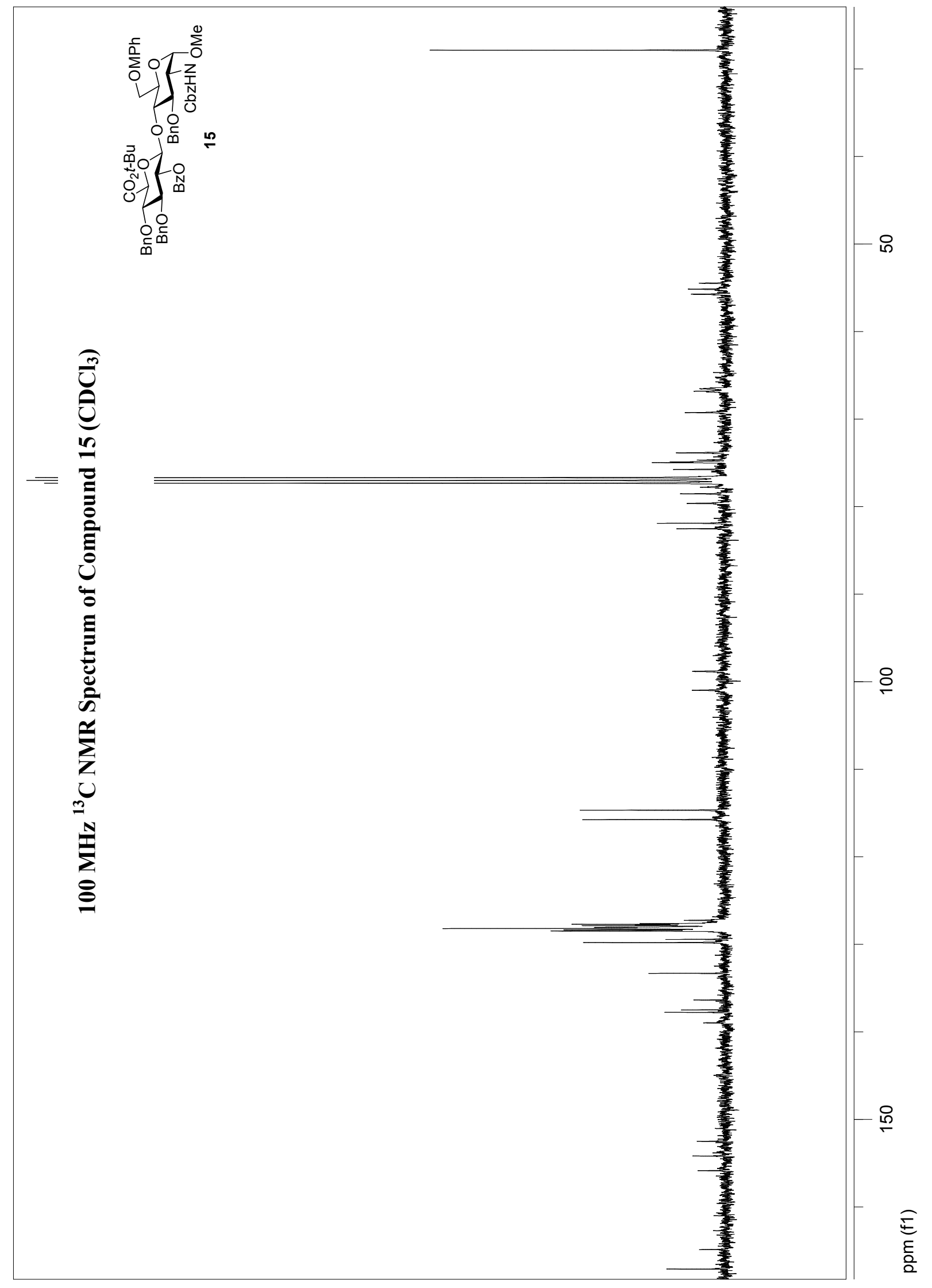


HSQC Spectrum of Compound $15\left(\mathrm{CDCl}_{3}\right)$
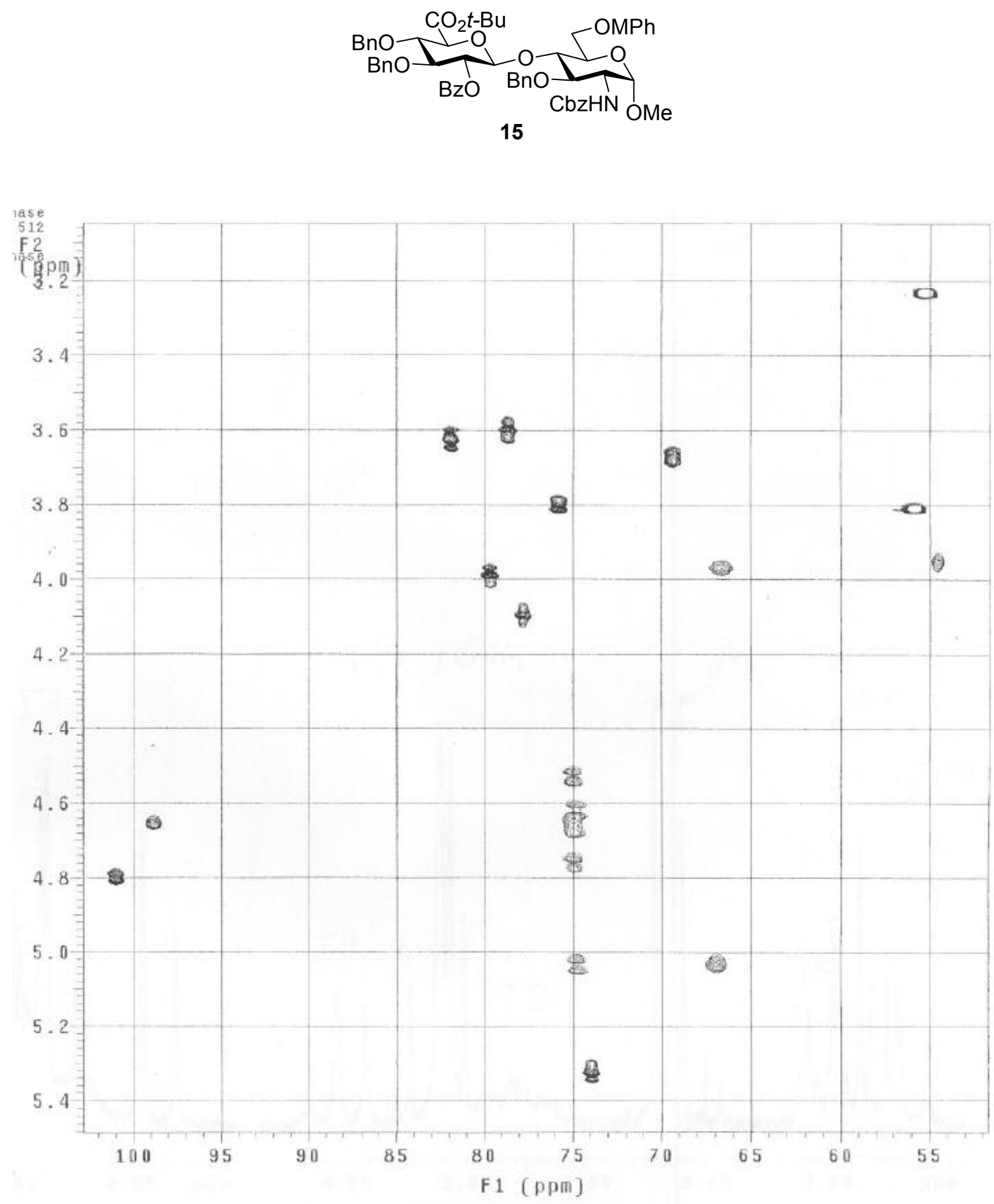


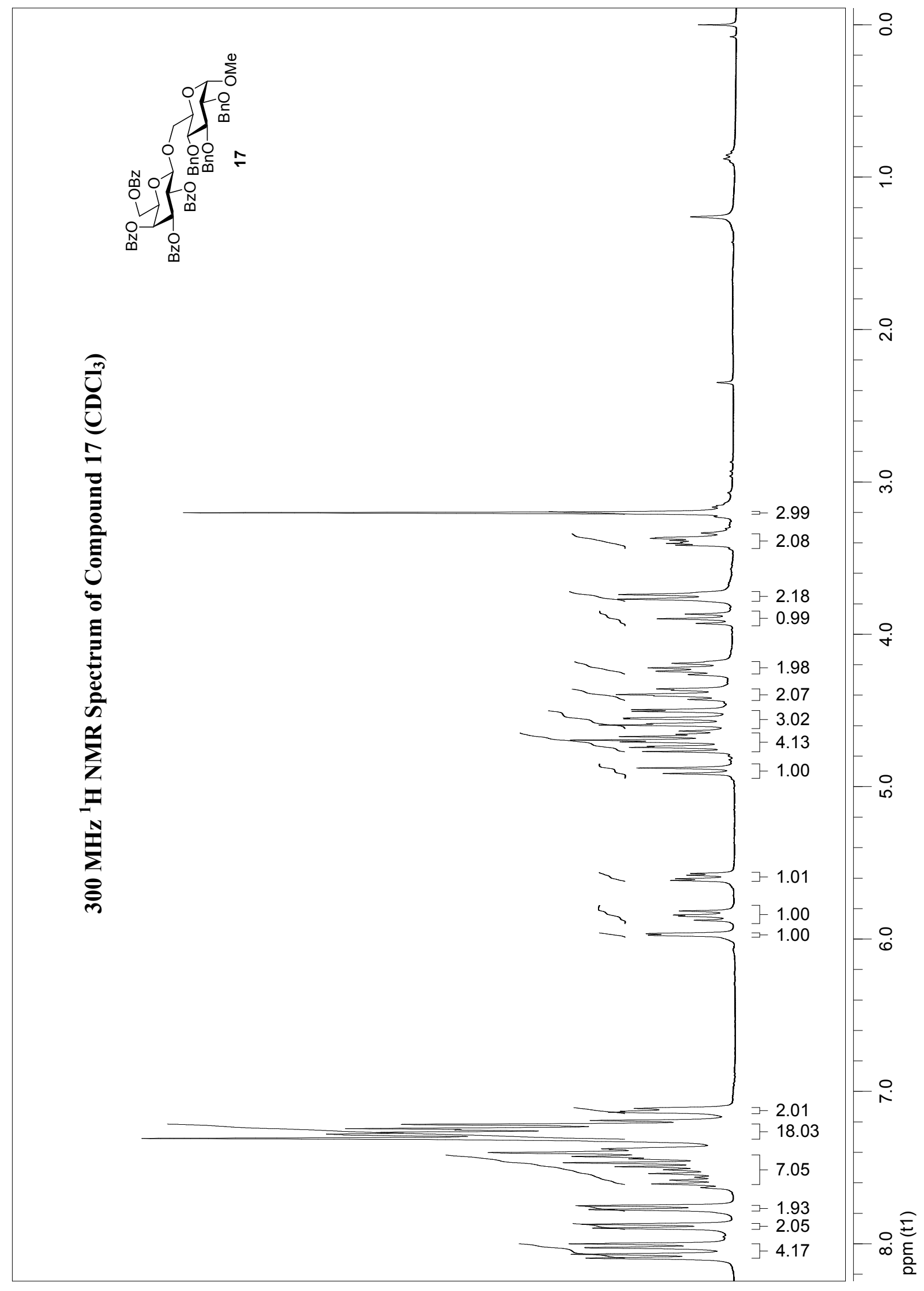




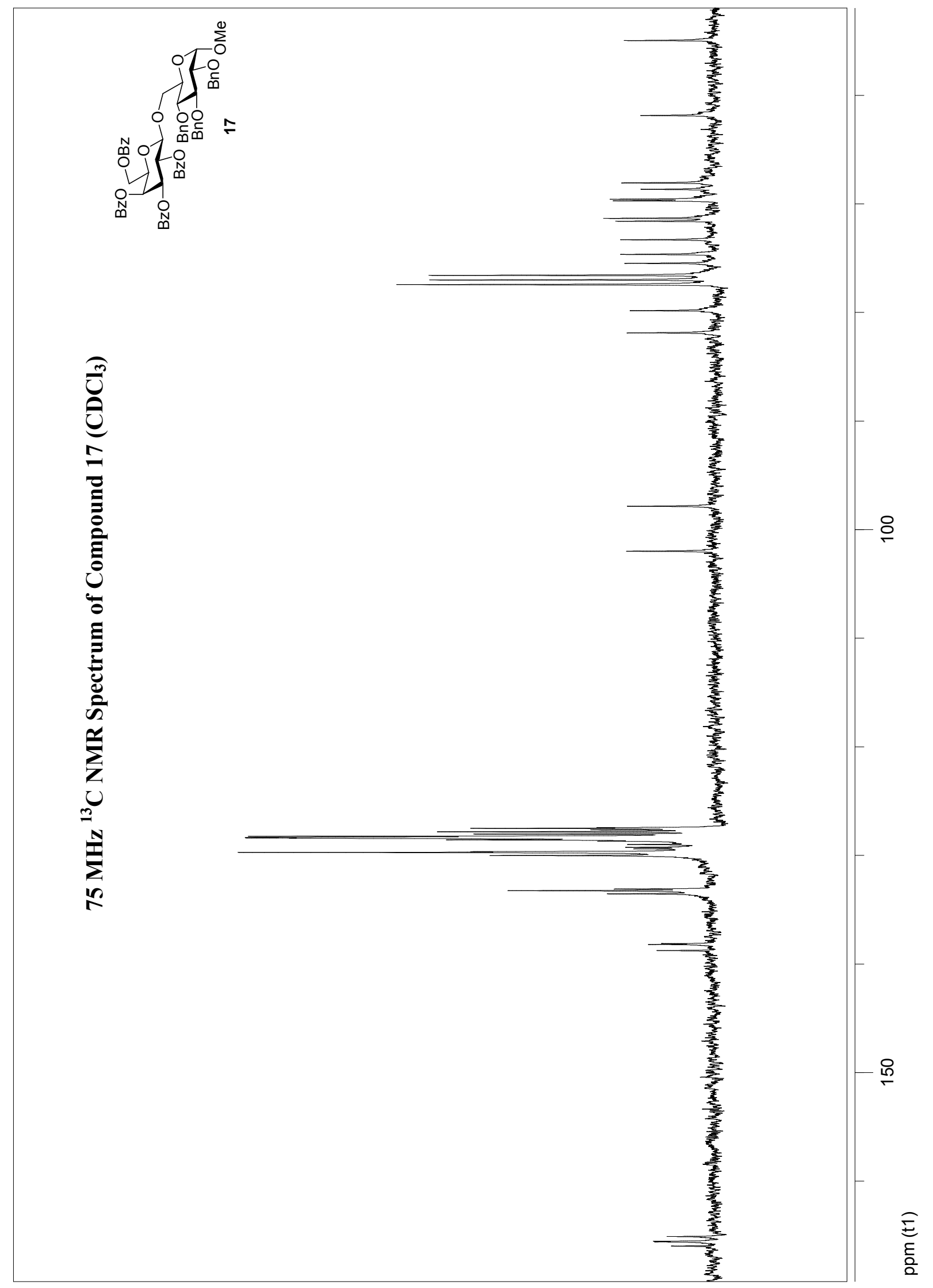


$300 \mathrm{MHz}$ COSY Spectrum of Compound $17\left(\mathrm{CDCl}_{3}\right)$
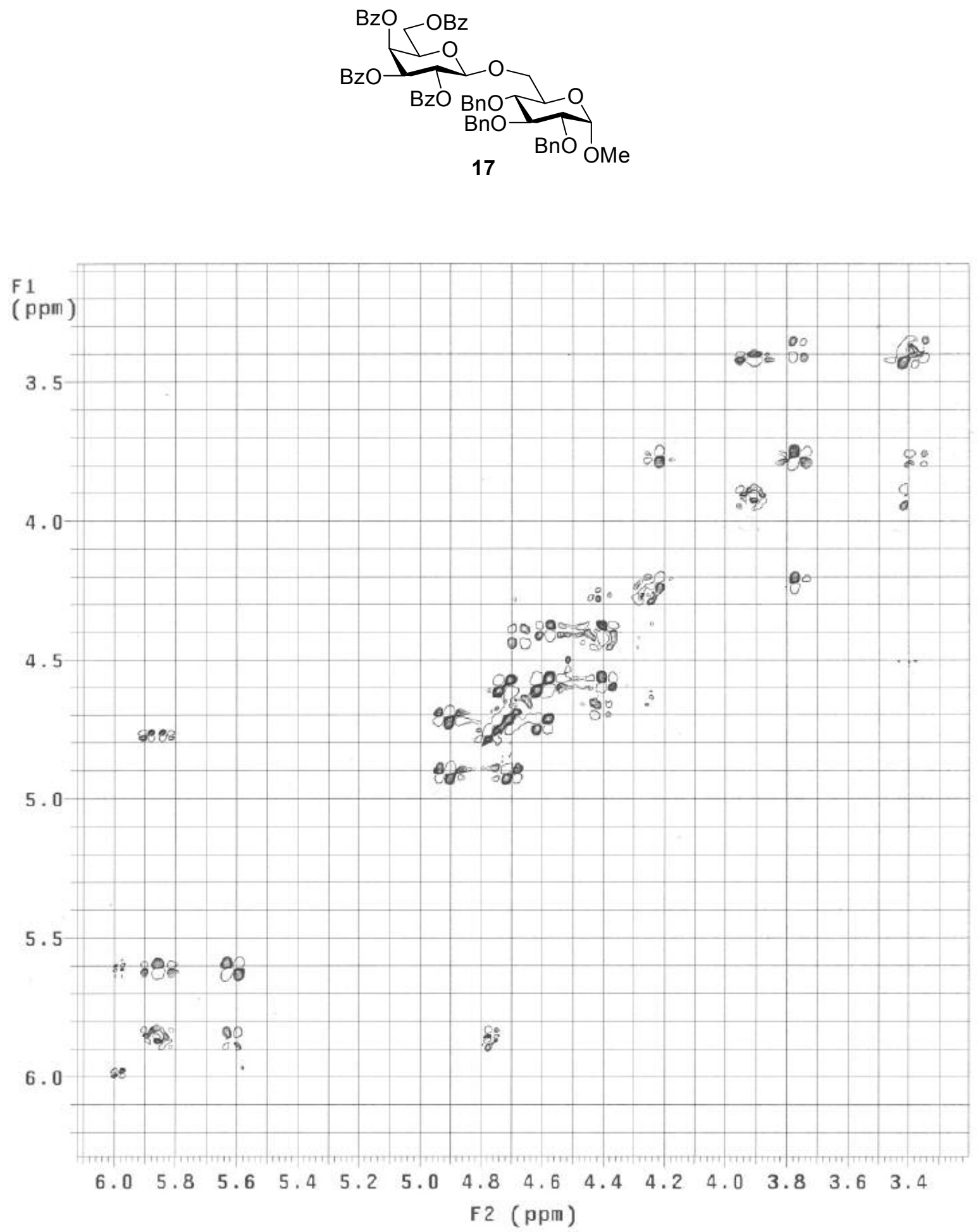
HSQC Spectrum of Compound $17\left(\mathrm{CDCl}_{3}\right)$
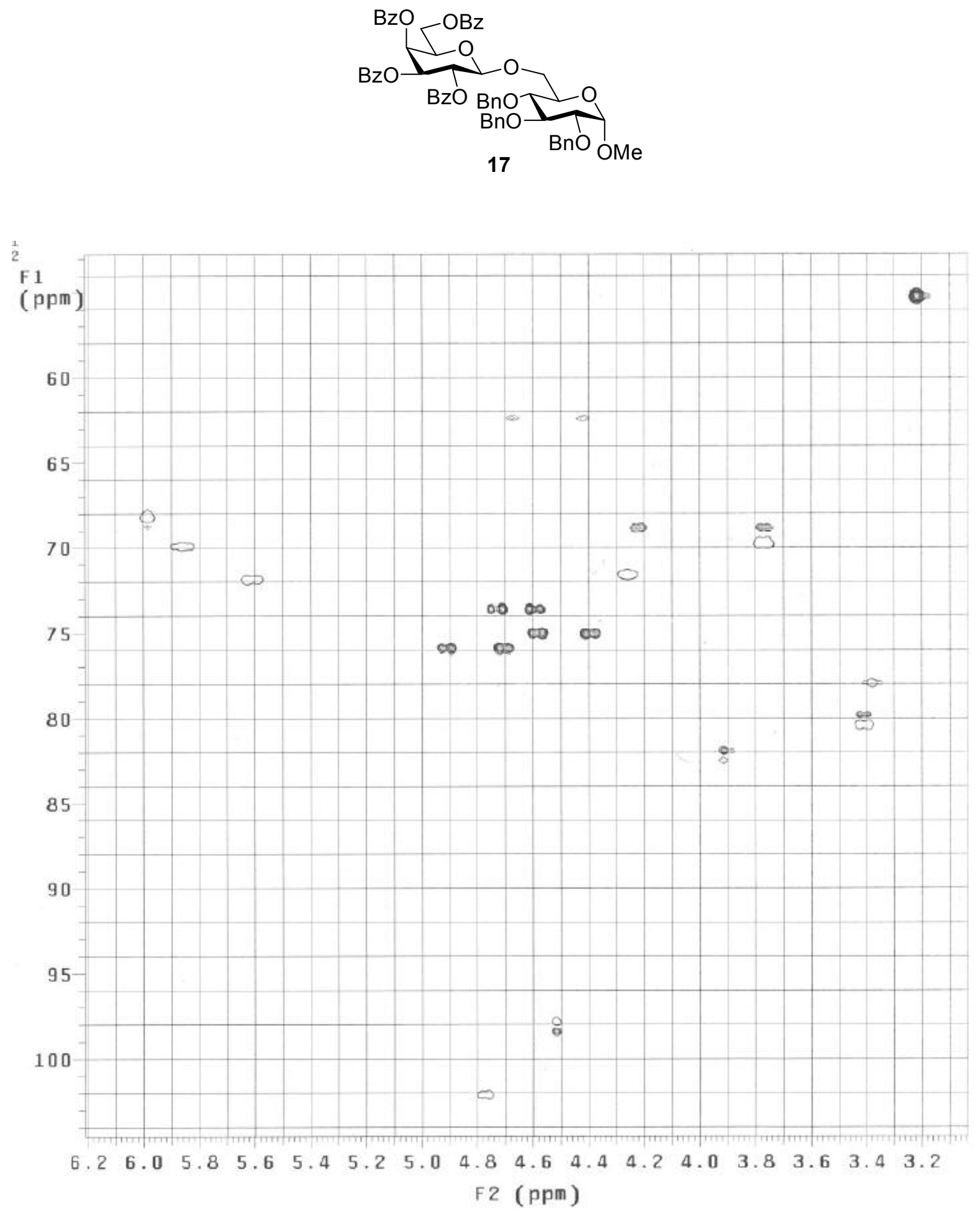


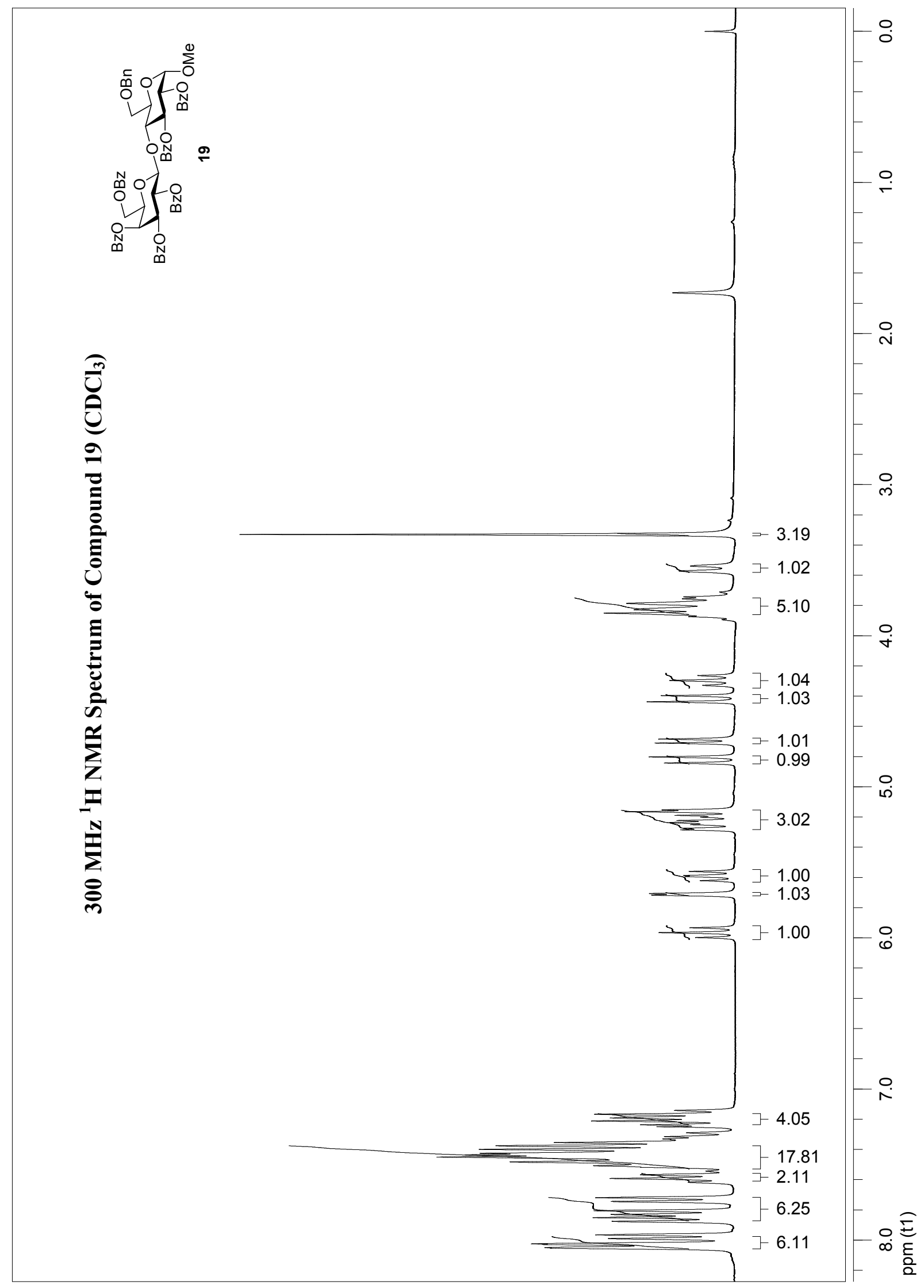




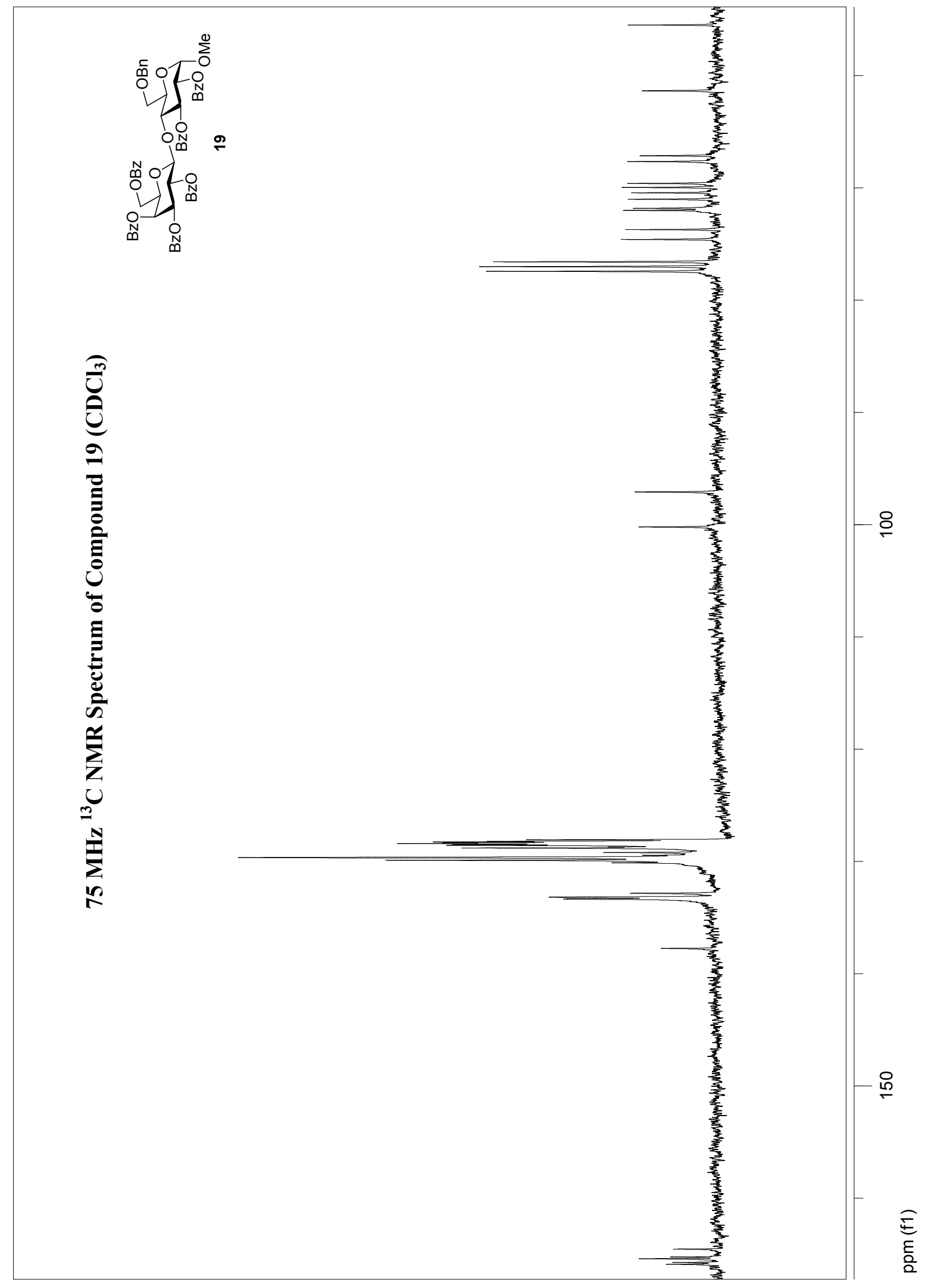


$300 \mathrm{MHz}$ COSY Spectrum of Compound $19\left(\mathrm{CDCl}_{3}\right)$

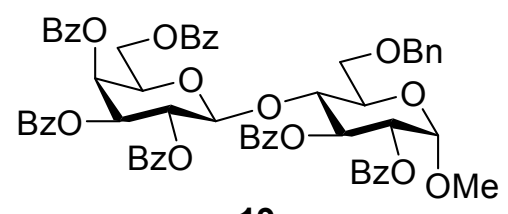

19

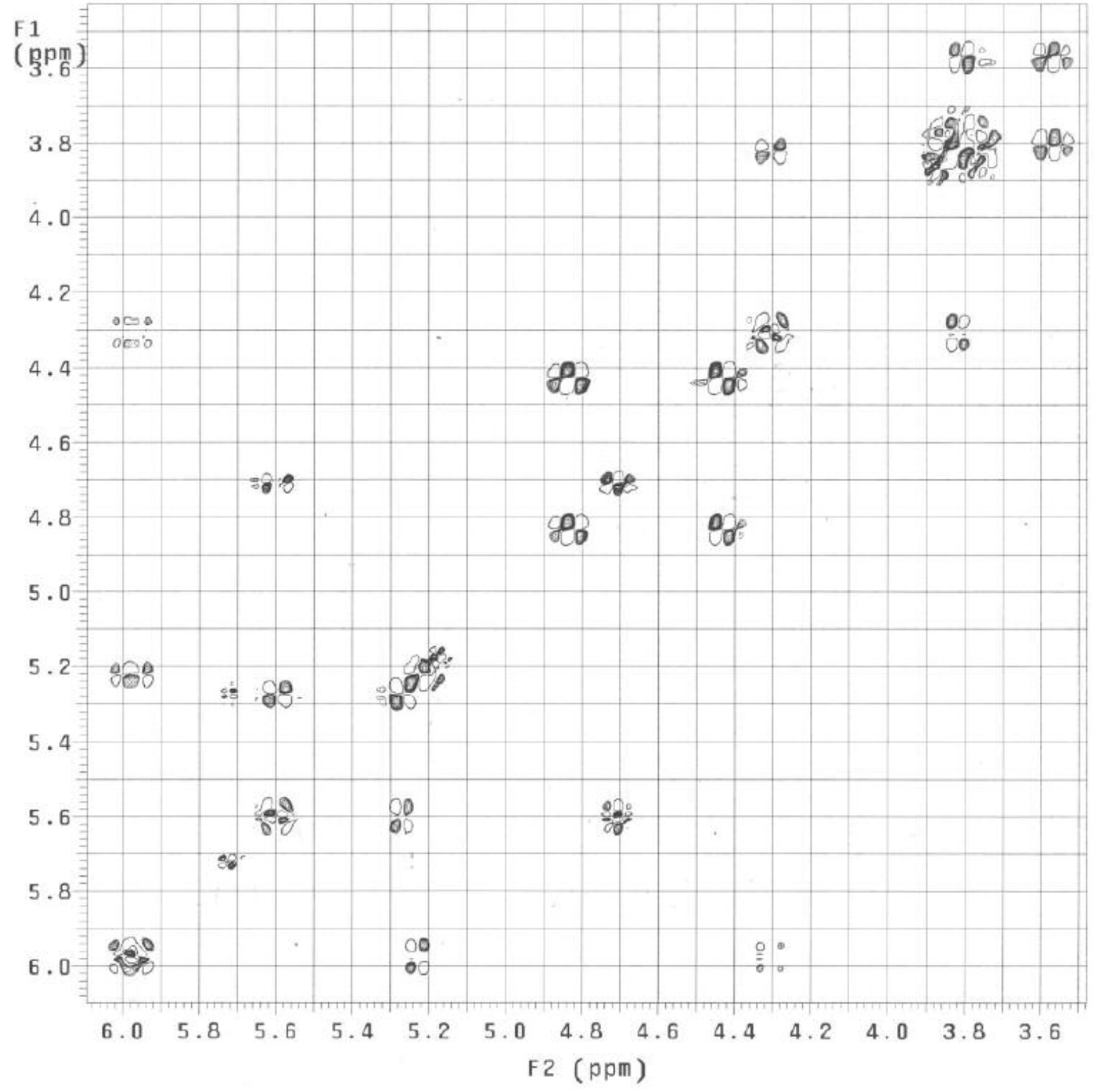


HSQC Spectrum of Compound $19\left(\mathrm{CDCl}_{3}\right)$
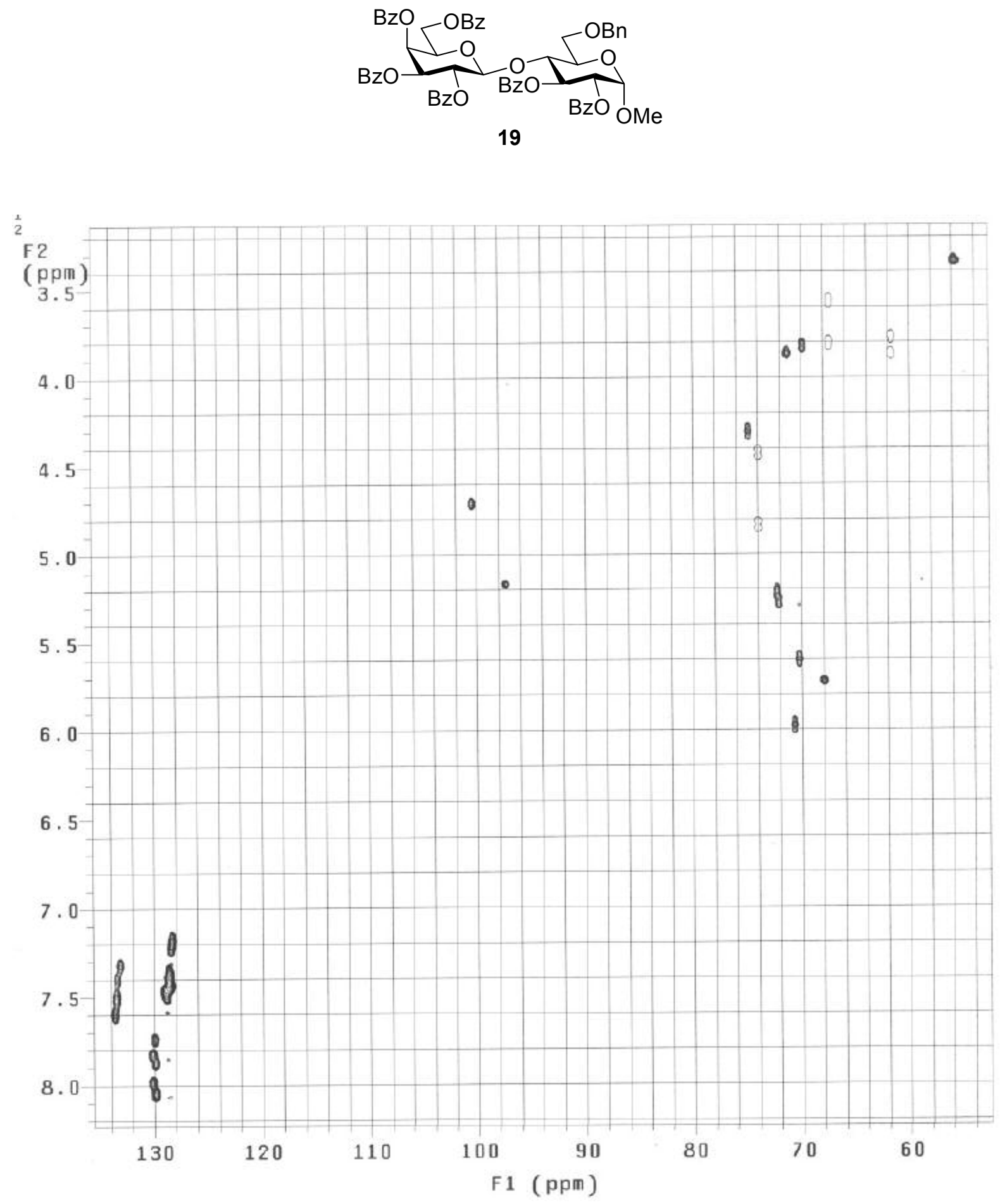


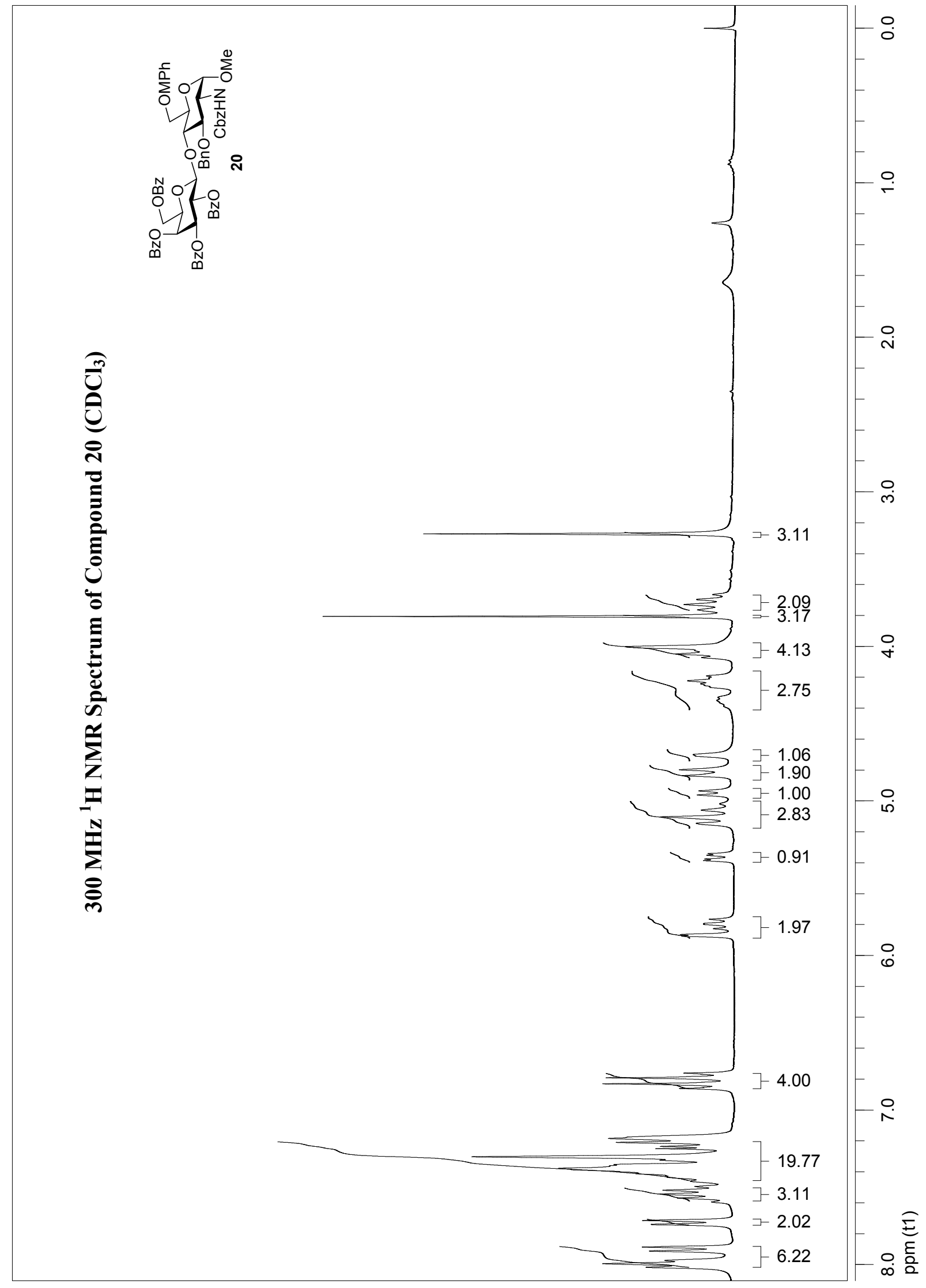




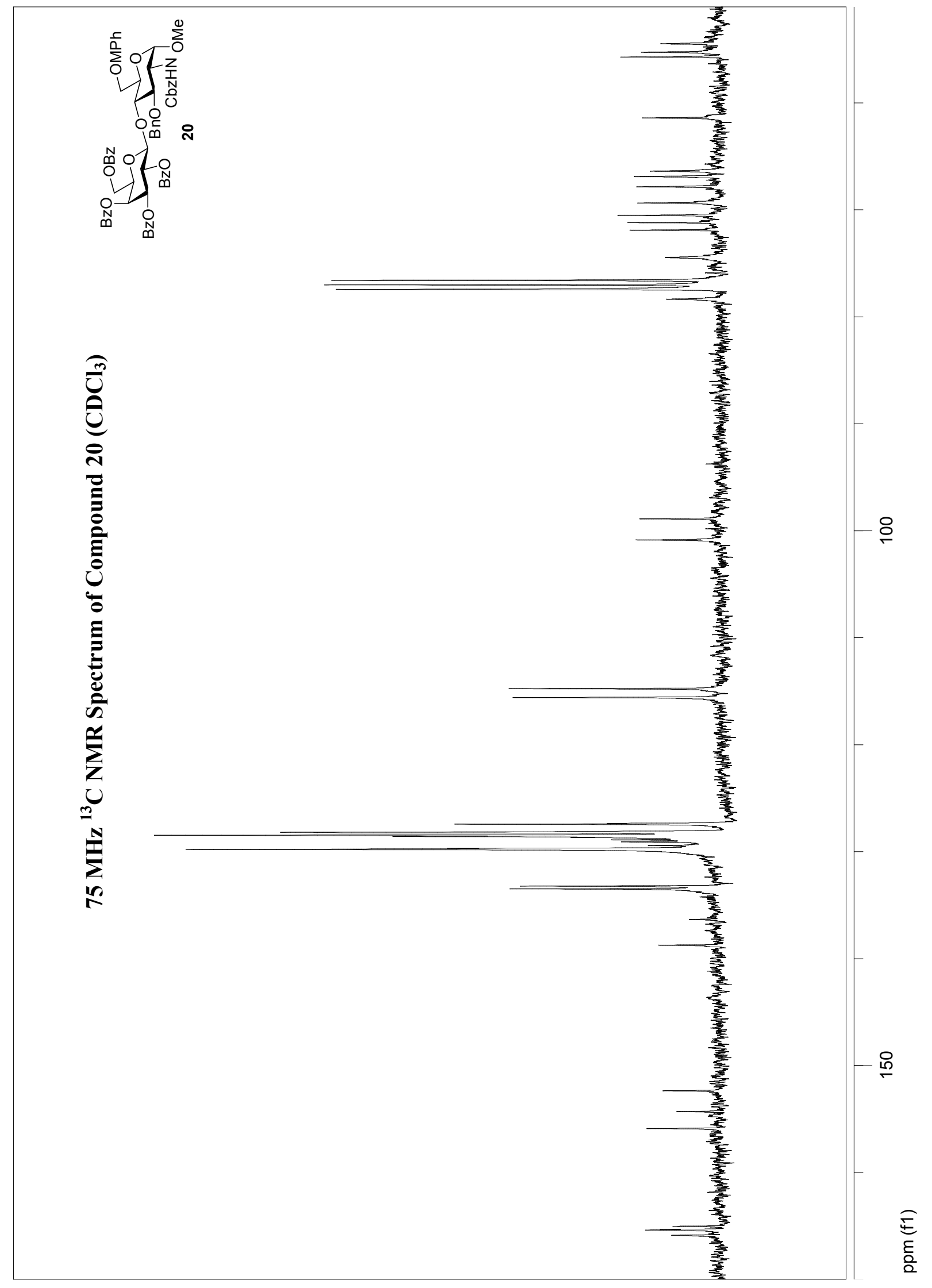


$300 \mathrm{MHz}$ COSY Spectrum of Compound $20\left(\mathrm{CDCl}_{3}\right)$
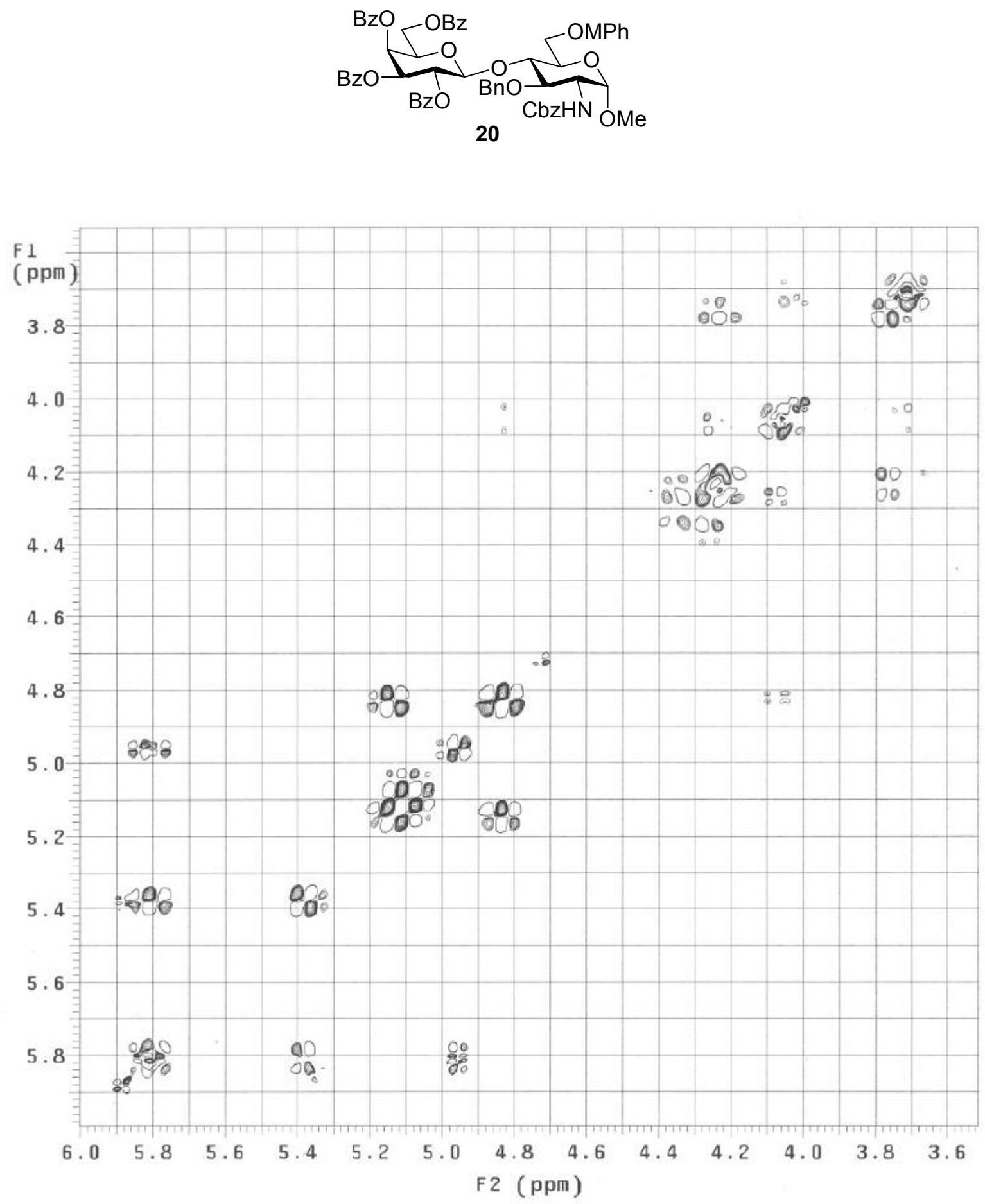
HSQC Spectrum of Compound $20\left(\mathrm{CDCl}_{3}\right)$
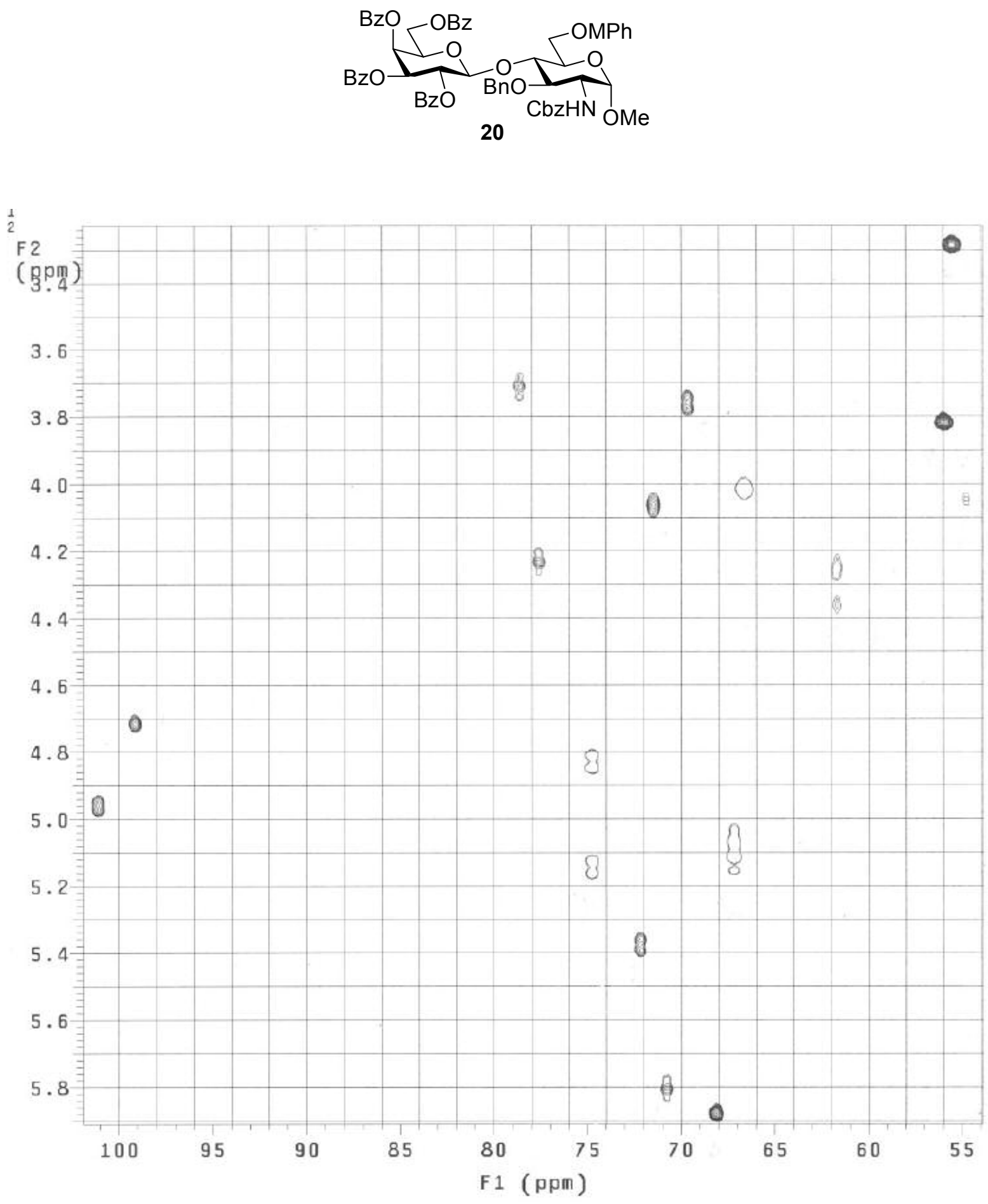


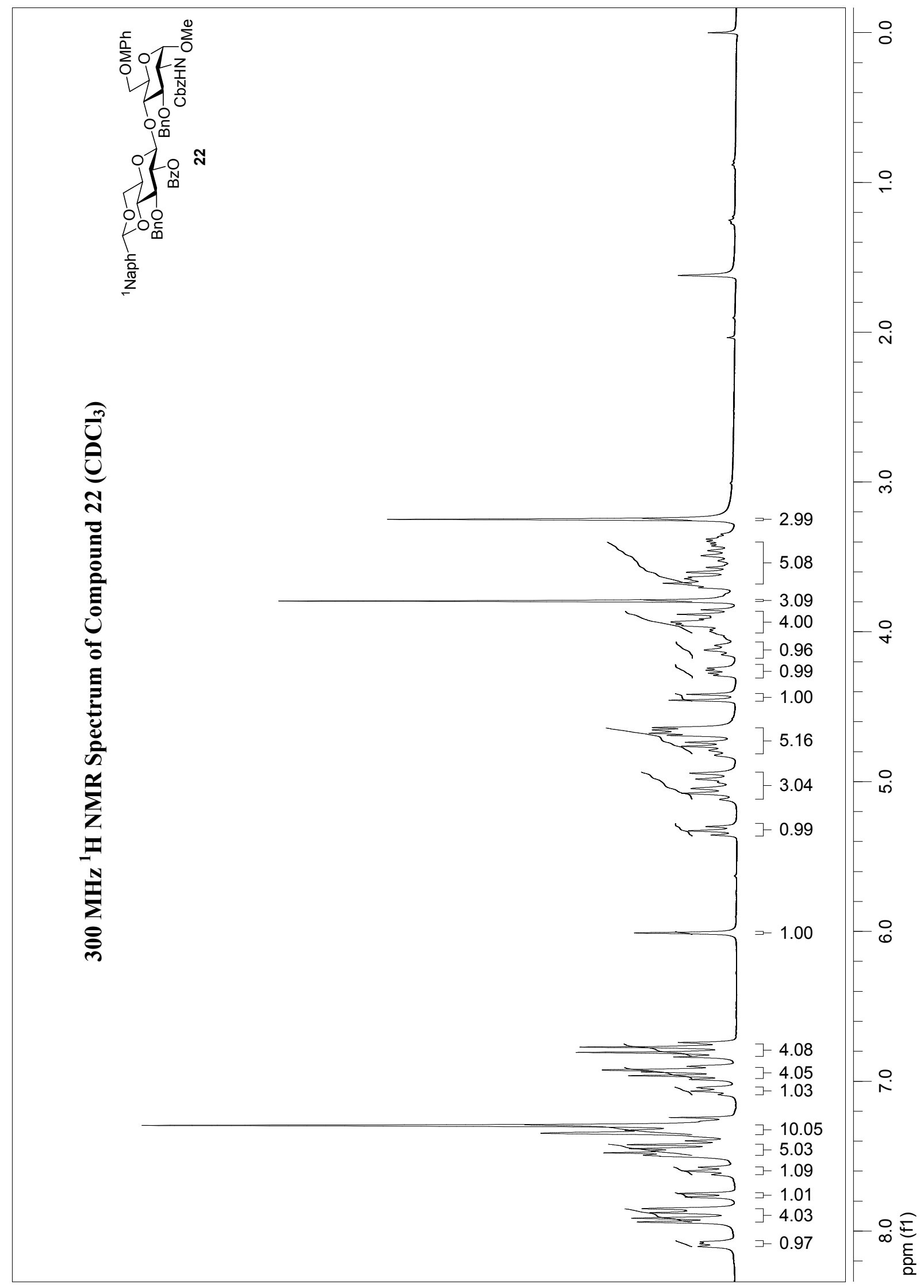




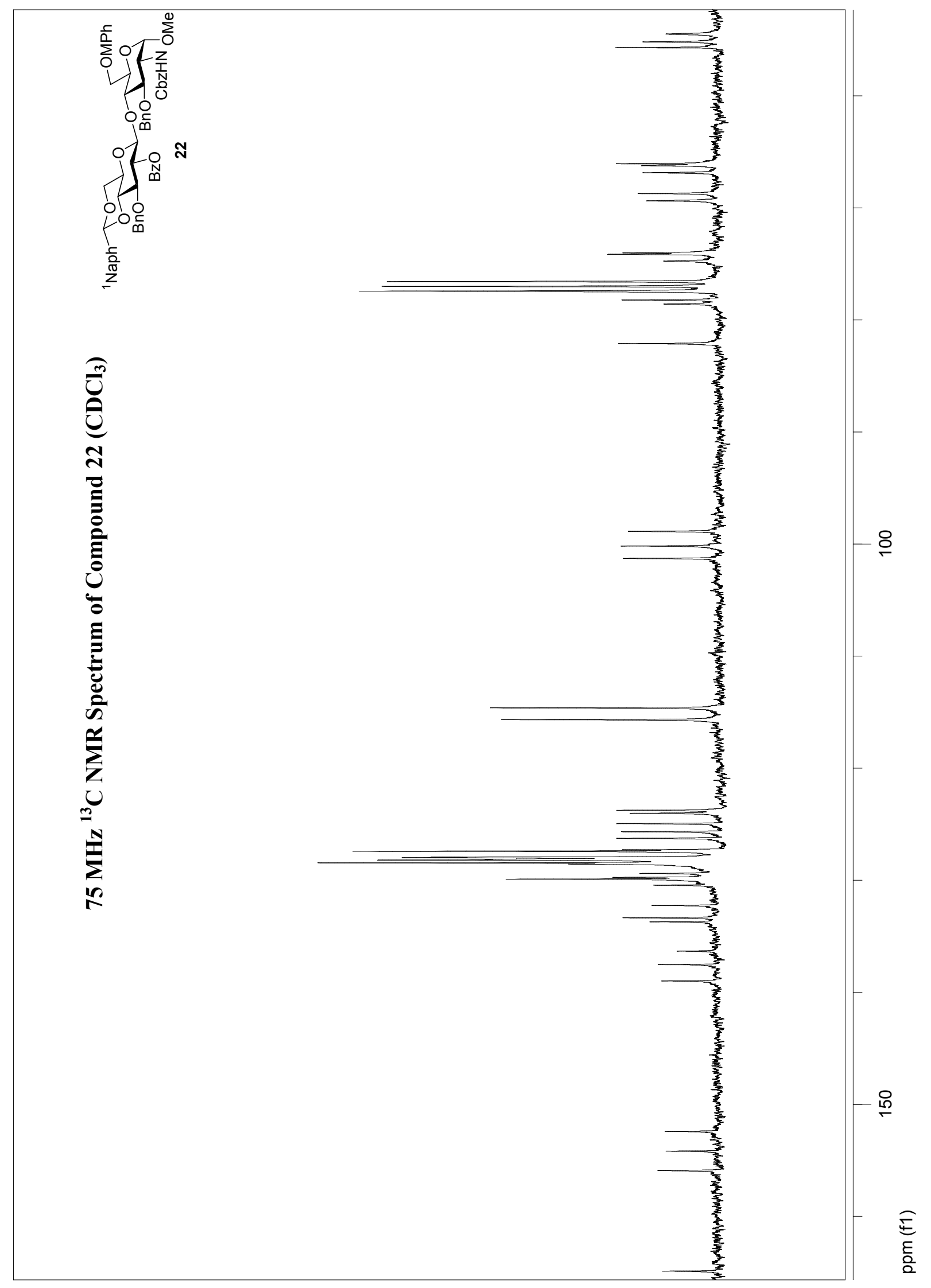


$300 \mathrm{MHz}$ COSY Spectrum of Compound $22\left(\mathrm{CDCl}_{3}\right)$

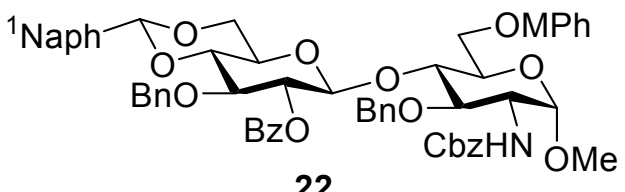

22

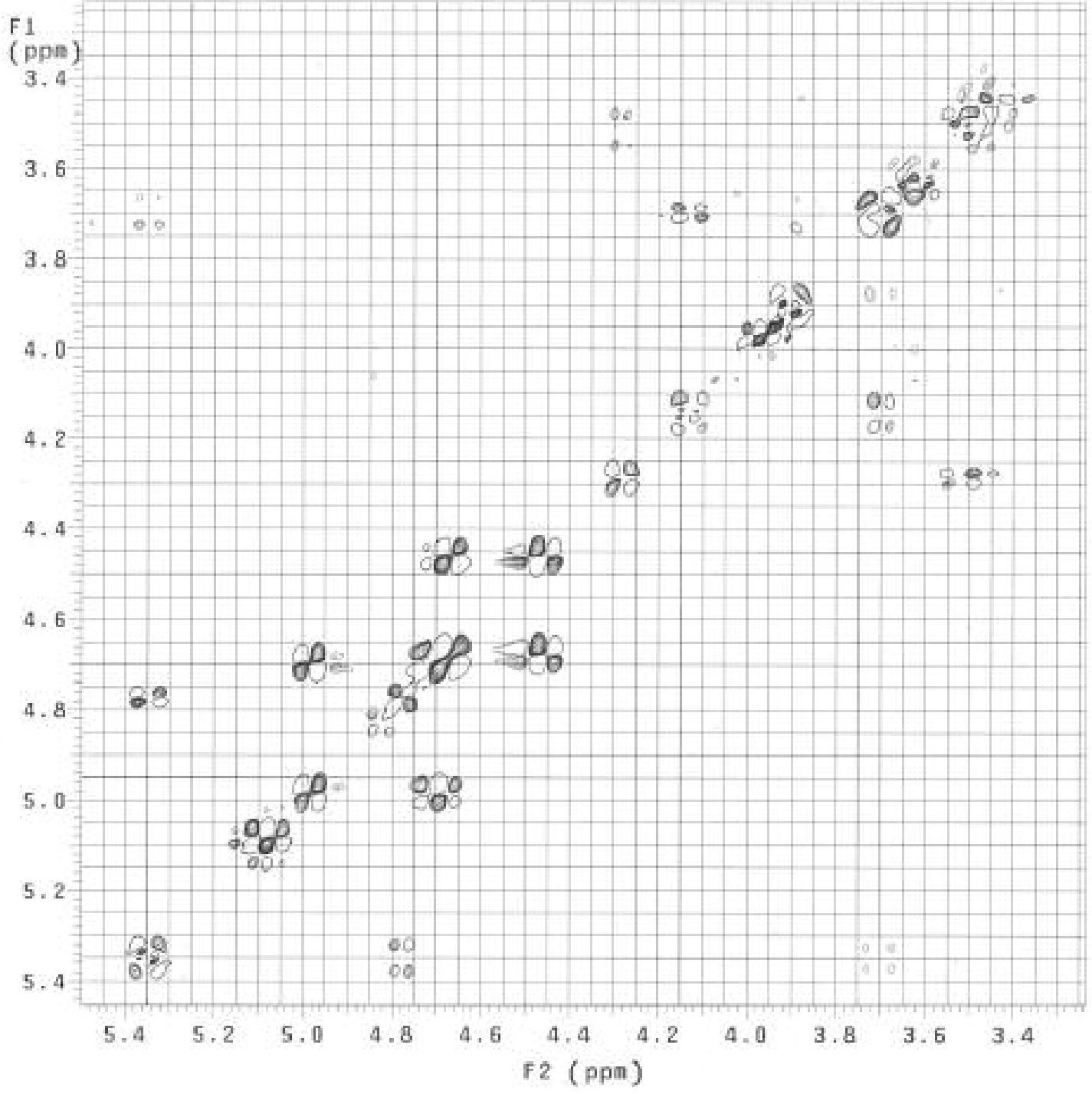


HSQC Spectrum of Compound $22\left(\mathrm{CDCl}_{3}\right)$
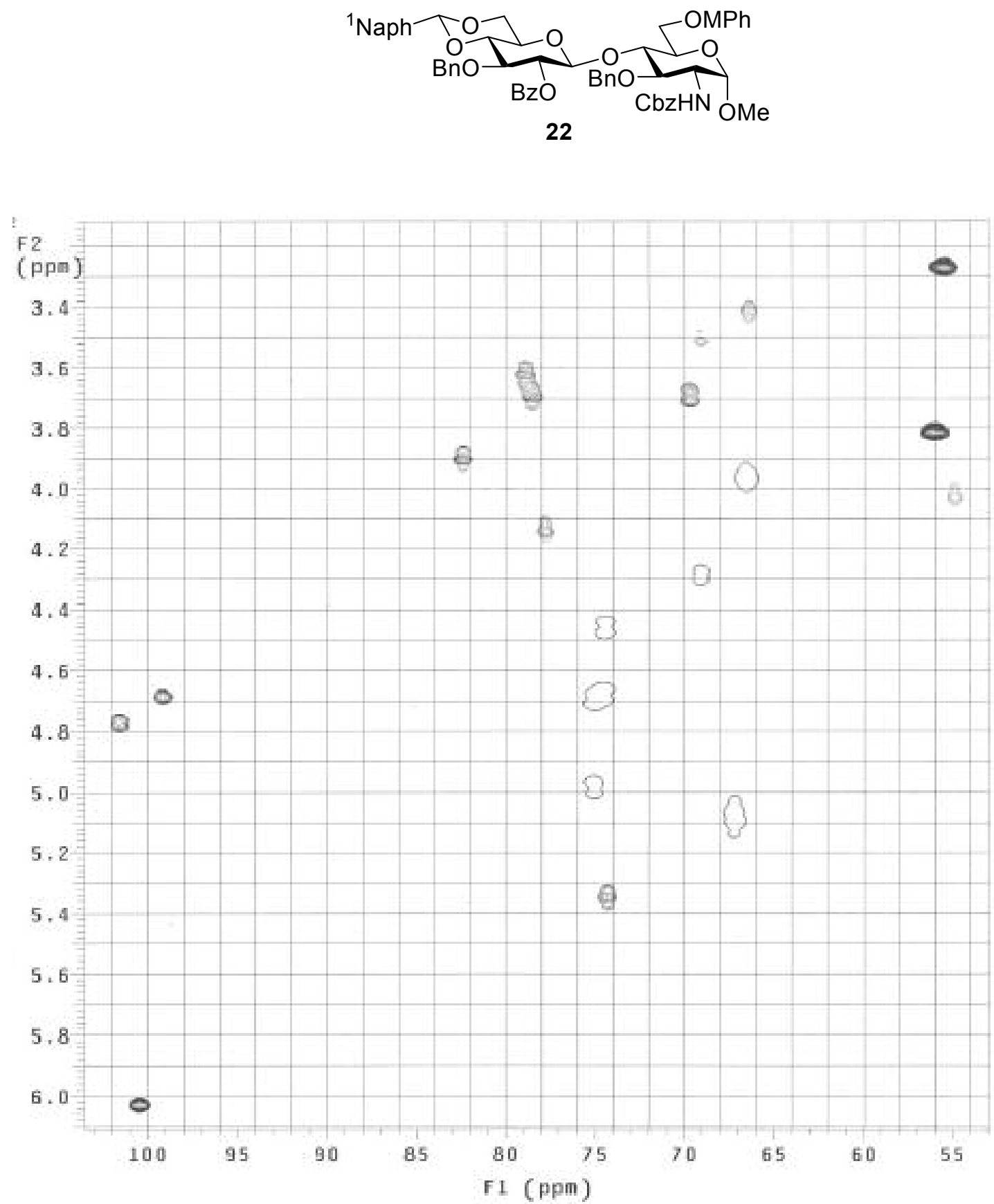


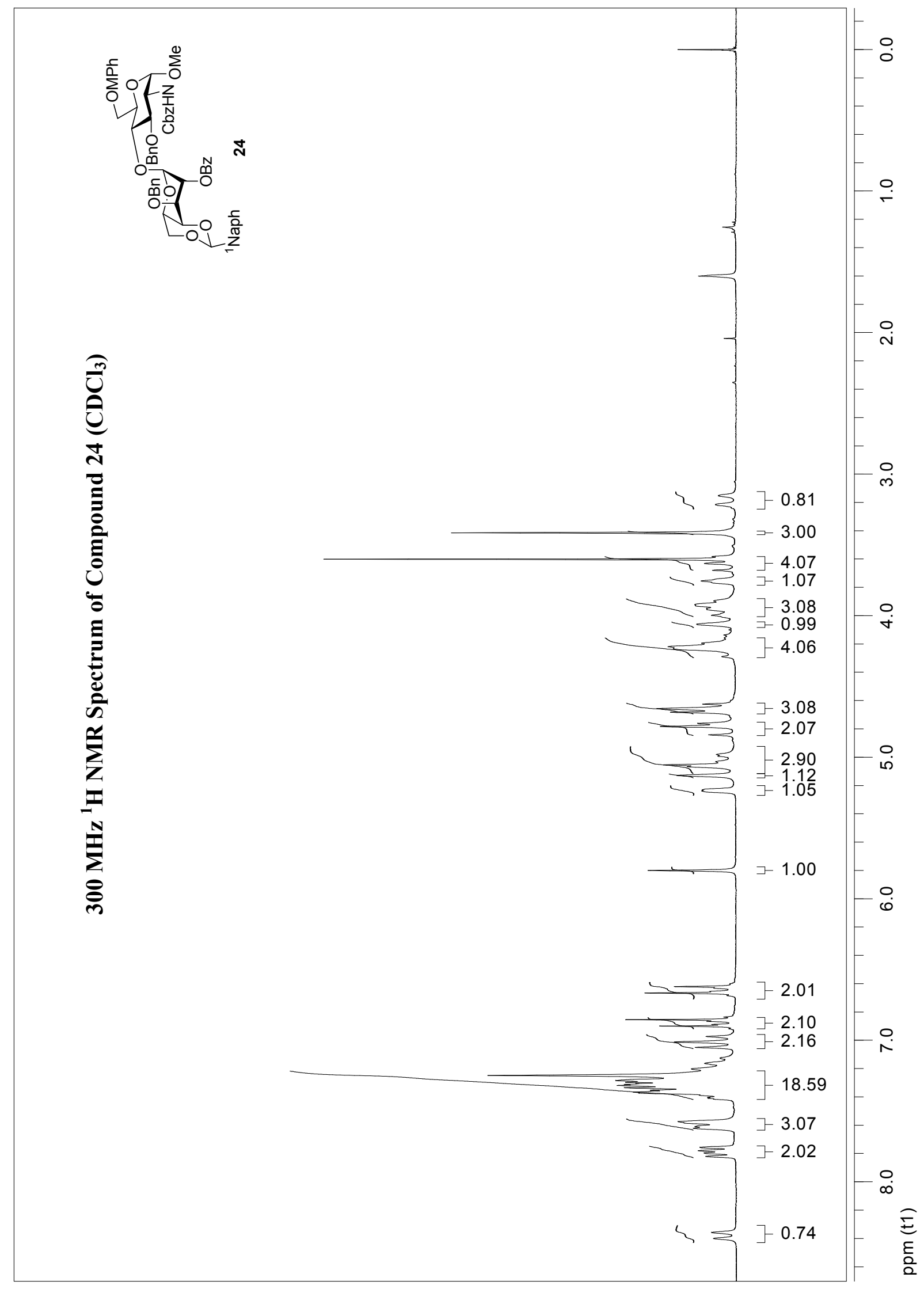




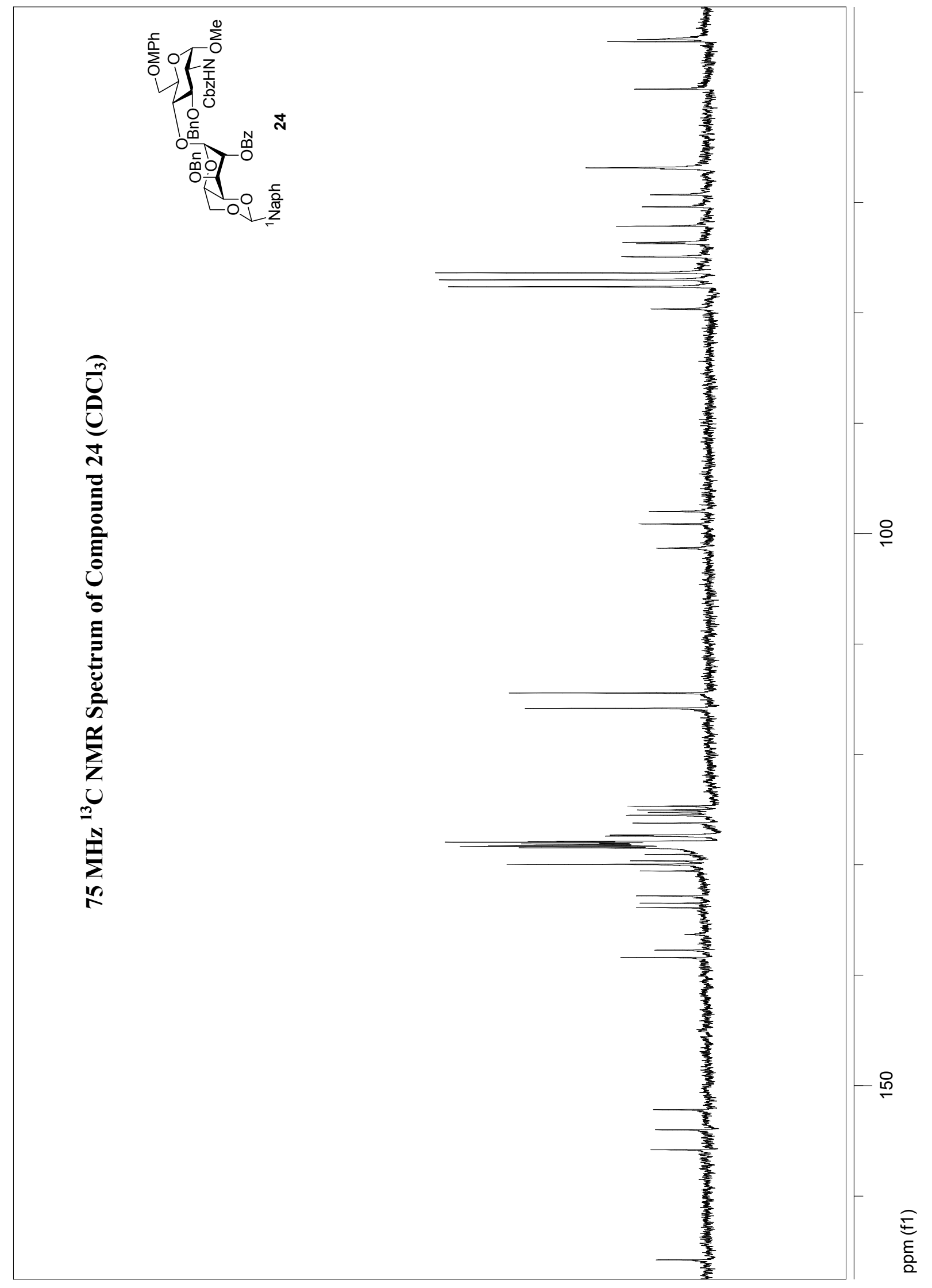


$300 \mathrm{MHz}$ COSY Spectrum of Compound $24\left(\mathrm{CDCl}_{3}\right)$
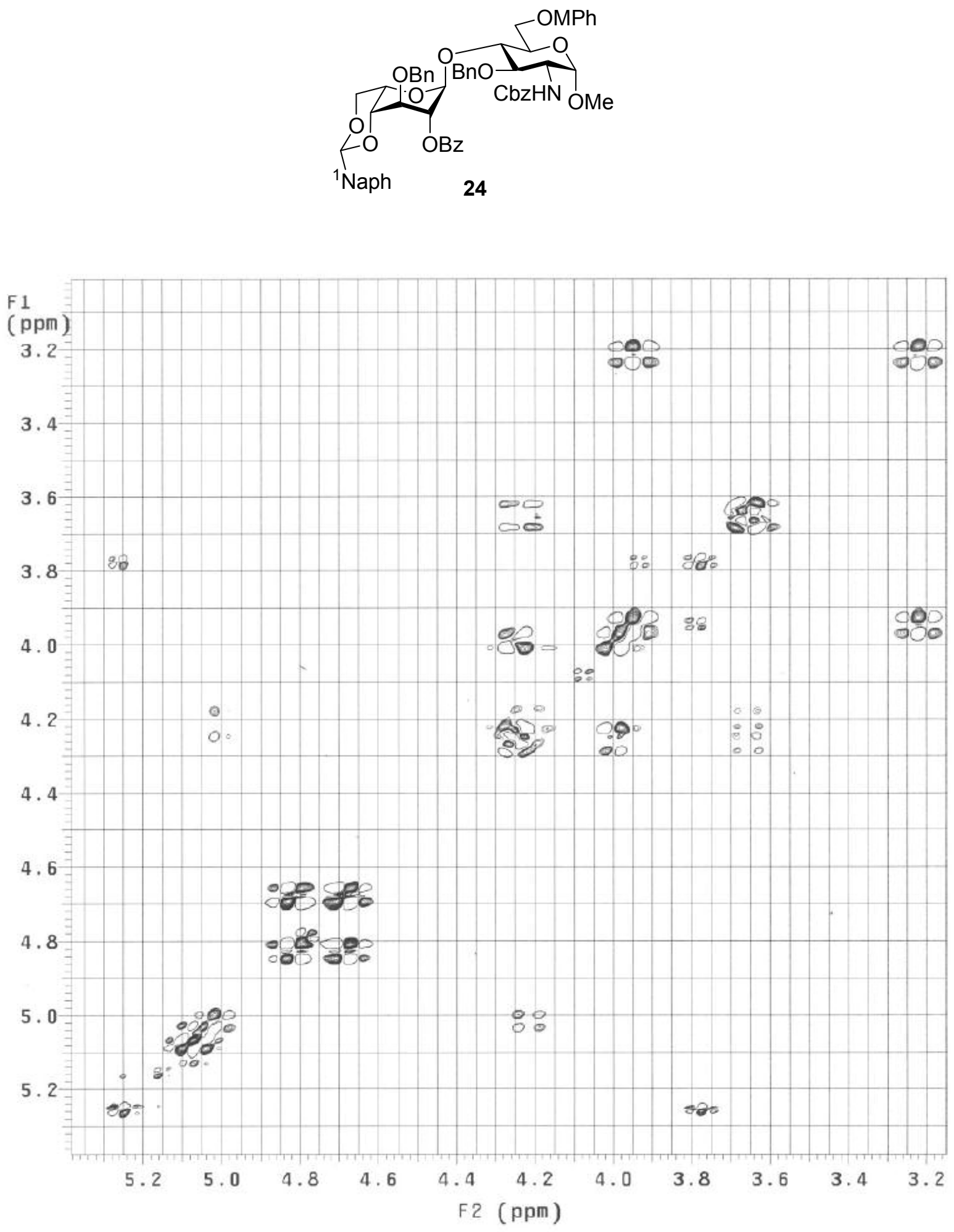
HSQC Spectrum of Compound $24\left(\mathrm{CDCl}_{3}\right)$
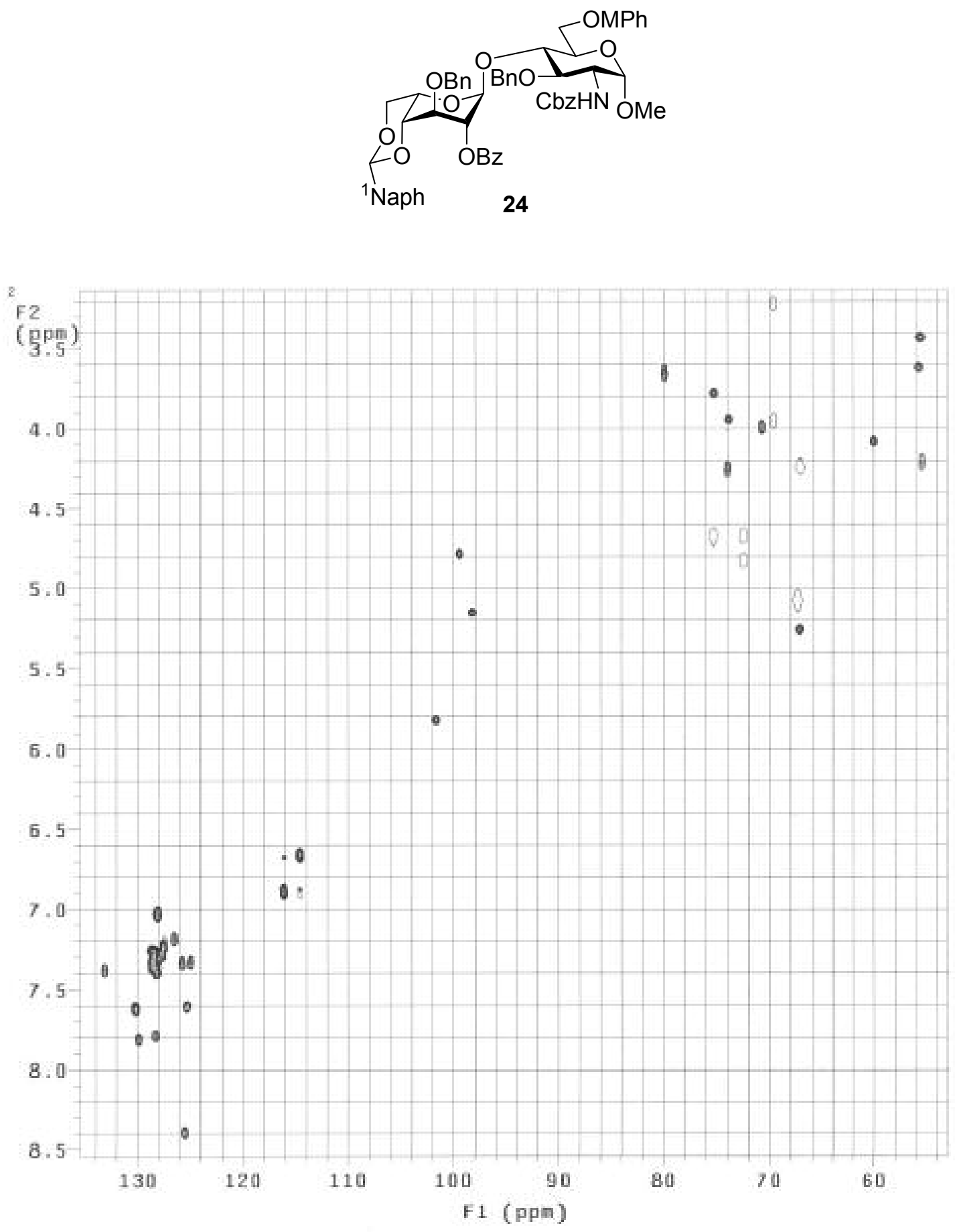